\title{
Deformed triangular lattice antiferromagnets in a magnetic field: Role of spatial anisotropy and Dzyaloshinskii-Moriya interactions
}

\author{
Christian Griset, ${ }^{1,2}$ Shane Head,${ }^{3}$ Jason Alicea,${ }^{4}$ and Oleg A. Starykh ${ }^{3}$ \\ ${ }^{1}$ Department of Physics, California Institute of Technology, Pasadena, California 91125, USA \\ ${ }^{2}$ Department of Physics, University of California, Santa Barbara, California 93106, USA \\ ${ }^{3}$ Department of Physics and Astronomy, University of Utah, Salt Lake City, Utah 84112, USA \\ ${ }^{4}$ Department of Physics and Astronomy, University of California, Irvine, California 92697, USA \\ (Received 7 July 2011; revised manuscript received 30 September 2011; published 12 December 2011)
}

\begin{abstract}
Recent experiments on the anisotropic spin-1/2 triangular antiferromagnet $\mathrm{Cs}_{2} \mathrm{CuBr}_{4}$ have revealed a remarkably rich phase diagram in applied magnetic fields, consisting of an unexpectedly large number of ordered phases. Motivated by this finding, we study the role of three ingredients-spatial anisotropy, Dzyaloshinskii-Moriya interactions, and quantum fluctuations-on the magnetization process of a triangular antiferromagnet, coming from the semiclassical limit. The richness of the problem stems from two key facts: (1) the classical isotropic model with a magnetic field exhibits a large accidental ground-state degeneracy and (2) these three ingredients compete with one another and split this degeneracy in opposing ways. Using a variety of complementary approaches, including extensive Monte Carlo numerics, spin-wave theory, and an analysis of Bose-Einstein condensation of magnons at high fields, we find that their interplay gives rise to a complex phase diagram consisting of numerous incommensurate and commensurate phases. Our results shed light on the observed phase diagram for $\mathrm{Cs}_{2} \mathrm{CuBr}_{4}$ and suggest a number of future theoretical and experimental directions that will be useful for obtaining a complete understanding of this material's interesting phenomenology.
\end{abstract}

DOI: 10.1103/PhysRevB.84.245108

PACS number(s): 75.10.Jm, 75.30.Ds, 75.50.Ee

\section{INTRODUCTION}

Antiferromagnetic spin models on the triangular lattice constitute one of the simplest and most widely studied realizations of geometric frustration. Indeed, the Ising triangular antiferromagnet was the first spin model found to possess a disordered ground state and extensive residual entropy ${ }^{1}$ at zero temperature $(T)$. While the classical Heisenberg model on the triangular lattice does order at $T=0$ into a well-known commensurate spiral pattern (also known as a $\sqrt{3} \times \sqrt{3}$ state), the fate of the quantum spin-1/2 Heisenberg Hamiltonian has been the subject of a long and fruitful debate spanning over 30 years of research. Although the originally proposed resonating valence bond liquid $^{2,3}$ did not emerge as the ground state of the spin-1/2 Heisenberg model, ${ }^{4-6}$ such a phase was later found in a related quantum dimer model on the triangular lattice. ${ }^{7}$

Triangular antiferromagnets in an applied magnetic fieldwhich is our focus here-have also been extensively studied for decades, and found to possess unusual magnetization physics that remains only partially understood. Underlying much of this interesting behavior is the discovery, made long ago, ${ }^{8}$ that in a magnetic field, Heisenberg spins with isotropic exchange interactions exhibit a large accidental classical ground-state degeneracy. That is, at finite magnetic fields, there exists an infinite number of continuously deformable classical spin configurations that constitute minimum energy states, but are in no way symmetry related. As reviewed later, this degeneracy is lifted by thermal (finite $T)^{8}$ and quantum (finite spin $\left.S\right)^{9}$ fluctuations; such fluctuation-driven selection, also known as order-by-disorder, ${ }^{10,11}$ results in a nontrivial temperature-field phase diagram ${ }^{8}$ consisting of three ordered phases. In particular, coplanar "Y" and "V" states are separated by a collinear up-up-down (UUD) phase, which realizes, for finite spin $S$ and at $T=0$, a one-third magnetization plateau over a finite field interval. This plateau state preserves continuous spin rotation symmetry about the magnetic field direction and is remarkably stable: unlike all other magnetically ordered states it survives "dimensional reduction" and exists even in the smallest possible triangular lattice strip, the two-chain zigzag ladder. ${ }^{12,13}$

To date a large number of magnetic materials have been synthesized that realize triangular antiferromagnets, and experiments on such compounds have highlighted the spectacular breadth of phenomena that can be driven by the interplay between magnetic fields and geometric frustration stemming from the lattice. For example, a stacked triangular antiferromagnet with weak interplane coupling is realized by the $S=5 / 2$ material $\mathrm{RbFe}\left(\mathrm{MoO}_{4}\right)_{2}$, whose phase diagram ${ }^{14}$ features all of the fluctuation-selected states discussed above. Reducing the magnitude of the magnetic ion's spin enhances quantum fluctuations, sometimes leading to highly nonclassical behavior. Low-spin materials that constitute deformed triangular antiferromagnets - that is, with spatially anisotropic exchange interactions that are not SU(2) symmetric-have indeed provided numerous surprises that are very likely quantum in origin. Studies of the spin-1/2 compounds $\mathrm{Cs}_{2} \mathrm{CuCl}_{4}$ and $\mathrm{Cs}_{2} \mathrm{CuBr}_{4}$ have been particularly fruitful in this regard. Inelastic neutron scattering has revealed striking dominance of a multiparticle continuum in the dynamical response of $\mathrm{Cs}_{2} \mathrm{CuCl}_{4} \cdot{ }^{15,16}$ This continuum is naturally explained in terms of spin-1/2 spinon excitations of weakly coupled chains. Ordered phases of this material show strong sensitivity to the magnitude and direction of the external magnetic field. ${ }^{17}$ The complexity of the $h-T$ phase diagram has been attributed to the competition between several asymmetric exchanges of the Dzyaloshinskii-Moriya (DM) type and a weak interplane exchange interaction. In particular, it has been suggested ${ }^{18}$ that, although weak, interplane exchange can dominate over stronger but frustrated interchain coupling and dictate the type of three-dimensional magnetic ordering at low temperatures.

Our study here is strongly motivated by the isostructural material $\mathrm{Cs}_{2} \mathrm{CuBr}_{4}$, whose triangular planes are less 
anisotropic and exhibit weaker interplane coupling compared to $\mathrm{Cs}_{2} \mathrm{CuCl}_{4}$, leading to rather different but equally rich phenomenology in magnetic fields. With fields directed in the plane of the triangular layers, the experimentally determined phase diagram for $\mathrm{Cs}_{2} \mathrm{CuBr}_{4}$ hosts as many as nine phases ${ }^{19}$ at low temperature. A one-third magnetization plateau features very prominently ${ }^{20-22}$ amongst the other, less understood, phases and offers a convenient starting point for theoretical analysis. ${ }^{23}$ Notably, $\mathrm{Cs}_{2} \mathrm{CuCl}_{4}$ shows no signs of this plateau, which has been attributed ${ }^{18}$ to its more pronounced spatial anisotropy and stronger interlayer coupling, ${ }^{24,25}$ which is known to suppress the plateau. ${ }^{26}$ And unlike the higherspin material $\mathrm{RbFe}\left(\mathrm{MoO}_{4}\right)_{2}$ discussed above, ${ }^{14}$ the width of the magnetization plateau in $\mathrm{Cs}_{2} \mathrm{CuBr}_{4}$ is essentially $T$ independent, ${ }^{21}$ strongly hinting at its quantum origin., ${ }^{9,23,27}$

The sheer number of states present in the phase diagram of $\mathrm{Cs}_{2} \mathrm{CuBr}_{4}$, in comparison with the three phases expected from the standard isotropic Heisenberg antiferromagnet on the triangular lattice, make it clear that a thorough theoretical study is required to begin understanding this material's complex behavior. Here, we attempt to access the global phase diagram of this system by analyzing the roles of three important known perturbations away from the "ideal" classical triangular antiferromagnet-quantum fluctuations stemming from the low-spin, $S=1 / 2$, spatial exchange anisotropy, and Dzyaloshinskii-Moriya (DM) interactions-all of which compete and favor different spin arrangements. Because the ideal classical model exhibits a large ground-state degeneracy, we show that even weak spatial anisotropy and DM coupling are sufficient to qualitatively alter the standard Y-UUD-V phase diagram, stabilizing new spin orders including incommensurate "umbrella" and planar states, a commensurate distorted-V spin structure, and a commensurate inverted-Y phase. Further experiments (such as neutron scattering and NMR measurements) will be very helpful for identifying which of these orders appear in the observed phase diagram, thereby sharpening the outstanding theoretical questions that undoubtedly remain. We also predict that-in sharp contrast to $\mathrm{Cs}_{2} \mathrm{CuCl}_{4}$-the phase diagram of $\mathrm{Cs}_{2} \mathrm{CuBr}_{4}$ ought to be insensitive to the direction of the magnetic field inside of the triangular planes.

The remainder of the paper is organized as follows. We provide an overview of the model and strategy that we pursue in Sec. II, and review the physics of the isotropic triangular antiferromagnet in Sec. III. Section IV addresses the case where DM coupling is present but spatial exchange anisotropy is neglected. We then explore the Bose-Einstein condensation of magnons near the saturation field in Sec. V, which allows us to simultaneously treat quantum fluctuations, DM coupling, and spatial anisotropy in a simple setting. The influence of spatial anisotropy on the global phase diagram is studied with and without DM interactions in Secs. VI and VII, respectively. Finally, we provide a summary and concluding remarks in Sec. VIII.

\section{MODEL AND STRATEGY}

We study the following Hamiltonian:

$$
H=\sum_{\left\langle\mathbf{r} \mathbf{r}^{\prime}\right\rangle} J_{\mathbf{r}, \mathbf{r}^{\prime}} \mathbf{S}_{\mathbf{r}} \cdot \mathbf{S}_{\mathbf{r}^{\prime}}-\sum_{r} \mathbf{h} \cdot \mathbf{S}_{\mathbf{r}}+H_{\mathrm{DM}},
$$

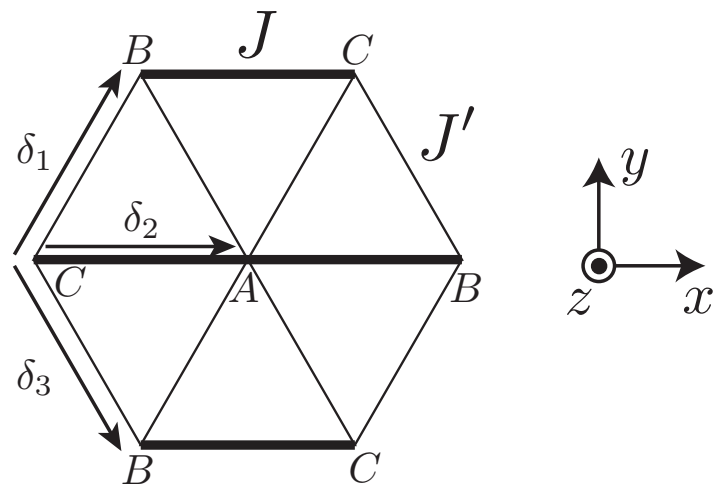

FIG. 1. Spatially anisotropic triangular lattice with exchange $J$ along horizontal bonds and $J^{\prime}$ along diagonal bonds. We define axes such that the sites lie in the $(x, y)$ plane as shown. Vectors $\boldsymbol{\delta}_{1,2,3}$ connect nearest-neighbor sites of the lattice. In the isotropic limit where $J=J^{\prime}$ and Dzyaloshinskii-Moriya coupling is absent, the Hamiltonian (1) exhibits a highly degenerate classical ground-state manifold wherein spins order in an underconstrained three-sublattice pattern. We label the three sublattices by $A, B$, and $C$.

where the exchange integral $J_{\mathbf{r}, \mathbf{r}^{\prime}}$ is given by $J$ on the horizontal bonds and $J^{\prime}$ on the diagonal zigzag bonds as shown in Fig. 1. For $\mathrm{Cs}_{2} \mathrm{CuBr}_{4}$, experiments have measured the values ${ }^{21}$ $J=11.3 \mathrm{~K}$ and $J^{\prime}=8.3 \mathrm{~K}$. The second term describes the Zeeman energy of spins in an external magnetic field while the third, $H_{\mathrm{DM}}$, represents the asymmetric DM interaction between neighboring spins.

Ideally, one would like to obtain the phase diagram for the quantum spin- $1 / 2$ problem above to begin understanding the interesting phenomenology of $\mathrm{Cs}_{2} \mathrm{CuBr}_{4}$. In this paper, we will attempt to access the physics of the spin- $1 / 2$ system coming from the large- $S$ limit, including quantum fluctuations perturbatively. This nevertheless still leaves a problem of substantial complexity, which can be understood by considering the classical, spatially isotropic limit of $H$, without DM coupling. Let us denote this minimal classical Hamiltonian by $H_{0}$. As noted in the introduction and reviewed in detail in Sec. III, at finite magnetic fields, $H_{0}$ exhibits a large "accidental" ground-state degeneracy. Quantum fluctuations (and thermal fluctuations at finite temperature) lift this groundstate degeneracy, as do spatial anisotropy and DM coupling. However, all of these ingredients compete with one another, favoring completely different ordered states. To resolve this competition, our strategy will be to compute the lowest-order energy splittings for the degenerate ground states of $H_{0}$ coming from each effect. As we will see later, quantum fluctuations and DM interactions split this degeneracy already at first order, while spatial anisotropy achieves this only at second order. Thus despite the fact that in $\mathrm{Cs}_{2} \mathrm{CuBr}_{4}$ spatial anisotropy [as quantified by $\left.\left(J-J^{\prime}\right) \sim 0.3 J\right]$ is expected to greatly exceed the characteristic energy scales for DM interactions in the material, the two can in fact comparably influence the phase diagram.

An additional complication arises from the fact that crystal symmetry of $\mathrm{Cs}_{2} \mathrm{CuBr}_{4}$ permits several DM terms, ${ }^{18}$ which together break the SU(2) spin symmetry enjoyed by the exchange coupling down to a discrete subgroup. One might then expect the phase diagram to depend sensitively both on 
the polar angle that $\mathbf{h}$ makes with respect to the $z$ axis, normal to the triangular plane, and the azimuthal angle $\mathbf{h}$ makes in the $(x, y)$ plane. Such a highly anisotropic phase diagram indeed emerges in the more spatially anisotropic material ${ }^{17}$ $\mathrm{Cs}_{2} \mathrm{CuCl}_{4}$. In the limit of weak spatial anisotropy-which due to the relevance to $\mathrm{Cs}_{2} \mathrm{CuBr}_{4}$ is our main focus here-most of these DM couplings fortunately play an unimportant role. As justified in Appendix A, it indeed suffices to consider only

$$
H_{\mathrm{DM}}=-\sum_{\mathbf{r}} \mathbf{D} \cdot\left[\mathbf{S}_{\mathbf{r}} \times\left(\mathbf{S}_{\mathbf{r}+\delta_{1}}+\mathbf{S}_{\mathbf{r}+\delta_{3}}\right)\right],
$$

where the DM vector is $\mathbf{D}=D \hat{\mathbf{z}}$ (we assume $D>0$ throughout) and $\delta_{1,3}$ are vectors shown in Fig. 1 . The strength of this $\mathrm{DM}$ interaction is expected to be comparable to that in the isostructural material $\mathrm{Cs}_{2} \mathrm{CuCl}_{4}$, where ${ }^{15} \mathrm{D} \approx J / 20$.

Notice that at $\mathbf{h}=\mathbf{0}$, the model with the above DM coupling still exhibits a U(1) spin symmetry corresponding to global spin rotations about the $\mathbf{D}$ vector. This leads to a major simplification - the phase diagram of $H$ depends on the polar angle $\mathbf{h}$ makes with the $z$ direction but not on the azimuthal angle. All additional DM terms, which lower this symmetry, lift the accidental degeneracy of $H_{0}$ only at second order in their couplings, whereas $H_{\mathrm{DM}}$ above has a first-order effect; see Appendix A for details. We emphasize that a nontrivial consistency check emerges here regarding relevance of our results to experiments on $\mathrm{Cs}_{2} \mathrm{CuBr}_{4}$. Our approach postulates that the physics of this material can be accessed coming from the classical, isotropic model without $\mathrm{DM}$ coupling. If this postulate is correct, then contrary to $\mathrm{Cs}_{2} \mathrm{CuCl}_{4}$, the experimental phase diagram of $\mathrm{Cs}_{2} \mathrm{CuBr}_{4}$ should be qualitatively insensitive to rotations of the field about the $\hat{\mathbf{z}}$ direction. Recent experiments by Y. Takano and collaborators $^{28}$ have indeed shown that the low-temperature phase diagram of $\mathrm{Cs}_{2} \mathrm{CuBr}_{4}$ is qualitatively the same for fields oriented along the material's $\mathbf{b}$ and $\mathbf{c}$ axes ( $x$ and $y$ axes in our notation from Fig. 1). This finding lends strong experimental support to our approach.

\section{REVIEW OF THE ISOTROPIC MODEL: $J=J^{\prime}, D=0$}

\section{A. Classical ground states}

In the absence of DM coupling, the classical isotropic Heisenberg model, where spins are described as threedimensional (3D) unit vectors, is well known to exhibit a large "accidental" ground-state degeneracy in applied magnetic fields. ${ }^{8}$ The classical ground-state structure can be conveniently exposed by expressing the Hamiltonian (up to a constant) as

$$
H_{0}=\frac{J}{2} \sum_{\mathbf{r}}\left[\mathbf{M}_{\Delta}(\mathbf{r})-\frac{\mathbf{h}}{3 J}\right]^{2},
$$

where

$$
\mathbf{M}_{\Delta}(\mathbf{r})=\mathbf{S}_{\mathbf{r}}+\mathbf{S}_{\mathbf{r}+\delta_{1}}+\mathbf{S}_{\mathbf{r}+\boldsymbol{\delta}_{2}}
$$

represents the magnetization of an elementary triangle located at site r. Below the saturation field $h_{\text {sat }}^{0}=9 \mathrm{~J}$, it is clear from Eq. (3) that classical ground states satisfy the condition $\mathbf{M}_{\Delta}(\mathbf{r})=\mathbf{h} /(3 J)$ for all $\mathbf{r}$. States fulfilling this requirement exhibit a three-sublattice structure as shown in Fig. 1, whose spins $\mathbf{S}_{A, B, C}$ are determined from

$$
\mathbf{S}_{A}+\mathbf{S}_{B}+\mathbf{S}_{C}=\frac{\mathbf{h}}{3 J}
$$

The ground-state degeneracy follows immediately from Eq. (5), since the spins on the three sublattices are specified by a total of six angles, which are constrained by only three equations. Parametrizing the spins on sublattice $\alpha$ as

$$
\mathbf{S}_{\alpha}=\left(\sin \theta_{\alpha} \cos \phi_{\alpha}, \sin \theta_{\alpha} \sin \phi_{\alpha}, \cos \theta_{\alpha}\right),
$$

the angles must specifically satisfy

$$
\begin{gathered}
\frac{\mathbf{h} \cdot \hat{\mathbf{x}}}{3 J}=\sin \theta_{A} \cos \phi_{A}+\sin \theta_{B} \cos \phi_{B}+\sin \theta_{C} \cos \phi_{C}, \\
\frac{\mathbf{h} \cdot \hat{\mathbf{y}}}{3 J}=\sin \theta_{A} \sin \phi_{A}+\sin \theta_{B} \sin \phi_{B}+\sin \theta_{C} \sin \phi_{C}, \\
\frac{\mathbf{h} \cdot \hat{\mathbf{z}}}{3 J}=\cos \theta_{A}+\cos \theta_{B}+\cos \theta_{C} .
\end{gathered}
$$

Equations (7) allow one to, say, express $\phi_{B}, \phi_{C}$, and $\theta_{C}$ in terms of $\phi_{A}, \theta_{A}$, and $\theta_{B}$. Note, however, that classical ground states do not exist for all possible values of the latter angles.

At $\mathbf{h}=0$, where the classical Hamiltonian exhibits $\mathrm{O}(3)$ symmetry, this degeneracy is symmetry related: the three unconstrained angles reflect the arbitrariness of the plane in which the $120^{\circ}$ spiral orients and the freedom for rotating all spins by an arbitrary angle within that plane. Introducing a finite magnetic field reduces the spin symmetry down to U(1). The three free angles nevertheless persist, only one of which is now symmetry related (corresponding to global rotations of the spins about the magnetic field axis). Consequently, in finite magnetic fields below saturation, $H_{0}$ exhibits an accidental classical ground-state degeneracy specified by two continuously deformable angles. Figure 2 illustrates some important members of this ground-state manifold, which we will frequently refer to later on: (a) Y, (b) up-up-down (UUD), (c) V, (d) distorted V, (e) inverted Y, and (f) umbrella states.

\section{B. Symmetries of the classical ground states}

It is important to emphasize the symmetry distinction between the above phases. Because $\left\langle\mathbf{S}_{\mathbf{r}} \cdot \mathbf{h}\right\rangle$ is uniform in umbrella configurations, this order is exceptional among the classical ground states in that it preserves the original lattice translation symmetry [when followed by an appropriate global U(1) spin rotation]. All other classical ground states exhibit a nontrivial three-sublattice pattern of $\left\langle\mathbf{S}_{\mathbf{r}} \cdot \mathbf{h}\right\rangle$ and therefore spontaneously break discrete translation symmetry. UUD order is also exceptional-it alone preserves global U(1) spin rotations about the field axis and therefore does not break any continuous symmetry. Ultimately, the U(1) spin symmetry enjoyed by the UUD state permits the formation of a one-third magnetization plateau in this system. In a boson representation of the spins, umbrella order corresponds to a superfluid phase, UUD order is a solid phase, and all other classical ground states constitute supersolids. $^{29-31}$

These supersolids can be further distinguished by symmetry. Since in the $\mathrm{V}$ state spins on two sublattices, say $A$ and $B$, are parallel, this order is symmetric under spatial $\pi / 3$ rotations about a site on the $C$ sublattice. This can also be 
(a) Y state

(b) UUD state

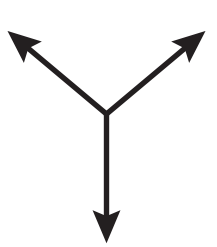

(d) distorted V

(e) inverted $Y$
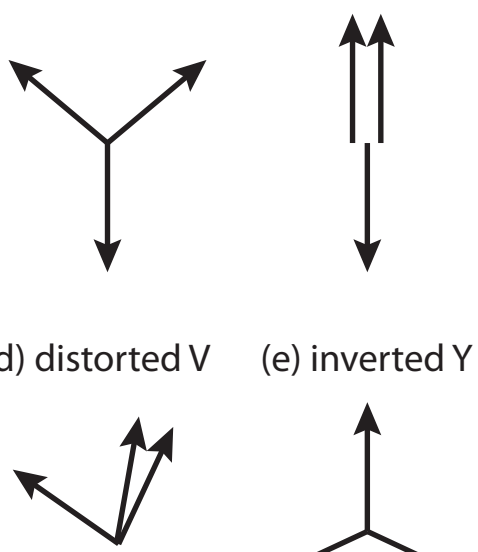

(c) V state

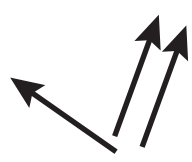

(f) umbrella

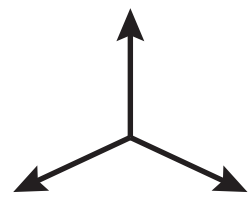

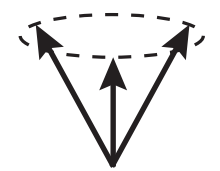

FIG. 2. Important members of the classical ground-state manifold exhibited by Eq. (1) when $J=J^{\prime}$ and Dzyaloshinskii-Moriya coupling is absent: (a) Y, (b) up-up-down (UUD), (c) V, (d) distorted $\mathrm{V}$, (e) inverted $\mathrm{Y}$, and (f) umbrella states. The arrows denote the spin orientations on the $A, B$, and $C$ sublattices of Fig. 1.

phrased as invariance of the $\mathrm{V}$ state with respect to permutation of the $A$ and $B$ sublattices. The distorted $\mathrm{V}$ state, while smoothly connected to the $\mathrm{V}$ state, violates this symmetry (the $A$ and $B$ sublattices are not equivalent anymore) and therefore constitutes a distinguishable phase in the isotropic system. Finally, in both the $\mathrm{Y}$ and inverted-Y states, spins on one sublattice, say $C$, point either along or against the field, and both orders are invariant under $\pi / 3$ rotations about a site on sublattice $C$ followed by a global $\pi$ spin rotation. While the latter phases exhibit identical symmetries, one can not deform the spins smoothly from one configuration to the other without breaking additional symmetries; thus it is still meaningful to classify them as different states.

All of the orders displayed in Fig. 2 thus constitute distinct phases when the full symmetries of the isotropic Heisenberg triangular antiferromagnet in field are present. Note, however, that when the symmetry is lowered by including DM coupling and/or spatial anisotropy, this ceases to be the case. The $\mathrm{V}$ and distorted-V states, for example, are not distinguishable once $\pi / 3$-rotation symmetry is removed by adding either of these ingredients. Similarly, the distinction between the UUD and Y states hinges on $U(1)$ spin symmetry and rotation symmetry, both of which are lost in the presence of DM coupling when the magnetic field has a finite component in the $(x, y)$ plane. The UUD and distorted-V states can, by contrast, remain distinct even in this physical situation, despite the very low symmetry remaining for the problem. For example, when $\mathbf{h}=h \hat{\mathbf{x}}$ and DM coupling is nonzero, the Hamiltonian remains invariant under $S_{\mathbf{r}}^{x} \rightarrow S_{-\mathbf{r}}^{x}$ and $S_{\mathbf{r}}^{y, z} \rightarrow-S_{-\mathbf{r}}^{y, z}$; the UUD state respects this discrete symmetry while the distorted-V state does not. We will return to these issues in subsequent sections of the paper.

\section{Influence of thermal fluctuations}

Thermal fluctuations provide one mechanism that lifts the large accidental ground-state degeneracy of the classical

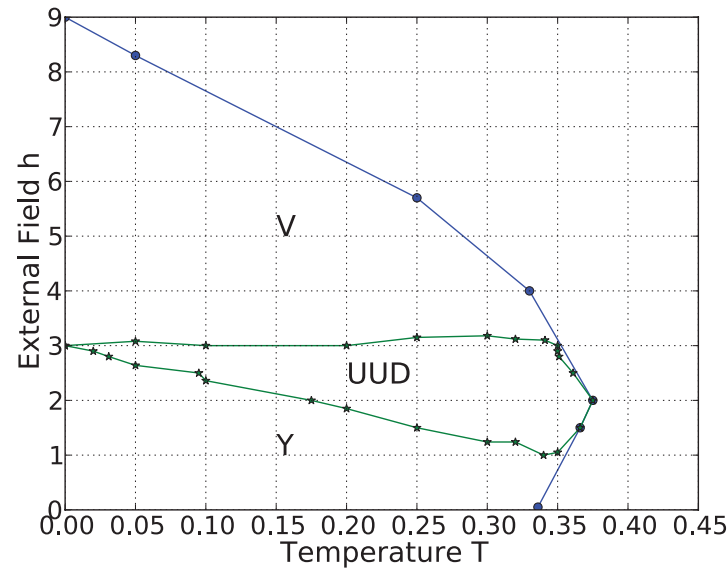

FIG. 3. (Color online) Phase diagram of the classical isotropic model. Amongst the large set of accidentally degenerate classical ground states, thermal fluctuations select the Y, UUD, and V states illustrated in Figs. 2(a) through 2(c).

model. At finite temperature the system minimizes its free energy, $F=U-T S$, and although the ground states exhibit identical energies $U$, their entropies generically differ. Thus only the most entropically favored states emerge. The finitetemperature phase diagram was established long ago, ${ }^{8}$ and is reproduced with our numerics in Fig. 3.

This set of Monte Carlo simulations is based on ALPS code. ${ }^{32}$ System sizes ranged from $24 \times 24$ to $96 \times 96$ with most simulations being carried out on $48 \times 48$ lattices. (The triangular lattice can be viewed as a square lattice with bonds along one diagonal; the system sizes above refer to the dimensions of such a square lattice. All simulations were performed with periodic boundary conditions.) Every $(T, h)$ coordinate was simulated using the standard Metropolis algorithm, with 500000 Monte Carlo steps for thermalization and another 500000 for measurement in the $24 \times 24$ system. For $48 \times 48$ lattices, these numbers were in the range 150000-250000. All Monte Carlo data presented here and below correspond to $J=1$. Different phases and boundaries between them have been identified by the behavior of the specific heat, the magnetization $M$, its field derivative $d M / d h$, and the vector chirality $\boldsymbol{\kappa}$ and coplanarity $K$ defined below.

The vector chirality is defined as

$\boldsymbol{\kappa}=\frac{2}{3 \sqrt{3}} \frac{1}{N} \sum_{\mathbf{r}}\left(\mathbf{S}_{\mathbf{r}} \times \mathbf{S}_{\mathbf{r}+\delta_{1}}+\mathbf{S}_{\mathbf{r}+\delta_{1}} \times \mathbf{S}_{\mathbf{r}+\delta_{2}}+\mathbf{S}_{\mathbf{r}+\delta_{2}} \times \mathbf{S}_{\mathbf{r}}\right)$,

where $N$ is the total number of spins in the system and the normalization factor ensures that the maximal chirality magnitude is unity. Taking $\mathbf{h}=h \hat{\mathbf{z}}$ for concreteness in the remainder of this section, it is useful to consider the longitudinal $\kappa_{z}$ and transverse $\kappa_{\perp}=\left(\kappa_{x}^{2}+\kappa_{y}^{2}\right)^{1 / 2}$ components of the vector chirality, in addition to its magnitude $\kappa=\left(\kappa_{z}^{2}+\kappa_{\perp}^{2}\right)^{1 / 2}$. Finite longitudinal chirality $\kappa_{z}$ identifies noncoplanar spin structures of umbrella type, while a nonzero transverse chirality signifies coplanar ordering in which no two spins in the unit cell are parallel (such as Y states). Notice that by construction, the chirality vanishes in three-sublattice states containing two parallel spins, such as the UUD and V states. 
The coplanarity $K$, introduced in Ref. 33, provides another useful indicator of planar spin structures. This quantity follows from the $A / B / C$ sublattice magnetizations

$$
\mathbf{M}_{A / B / C}=\frac{1}{N} \sum_{\mathbf{r} \in A / B / C} \mathbf{S}_{\mathbf{r}}
$$

by constructing

$$
\mathbf{K}_{A B}=\left(\mathbf{M}_{A} \times \mathbf{M}_{B}\right) \times \hat{\mathbf{h}},
$$

and similarly for $\mathbf{K}_{B C}$ and $\mathbf{K}_{C A}$. The coplanarity is then given by the combination

$$
K^{2}=\left|\mathbf{K}_{A B}\right|^{2}+\left|\mathbf{K}_{B C}\right|^{2}+\left|\mathbf{K}_{C A}\right|^{2} .
$$

As defined $K$ is finite for any coplanar spin configuration which includes the magnetic field in its plane. In particular, $K$ is finite in the $\mathrm{V}$ state but vanishes in the collinear UUD configuration.

Our Monte Carlo simulations, summarized in Fig. 3, agree well with existing data ${ }^{8,34}$ and clearly demonstrate entropic selection of the Y, UUD, and V states over noncoplanar order. This is consistent with the usual intuition that entropy disfavors noncoplanar structures and favors coplanar and, when available, collinear ordering. ${ }^{10,11}$ Coplanarity of the selected states is reflected in an exceedingly small value of the longitudinal chirality $\kappa_{z}$ over the entire magnetic field range $0 \leqslant h \leqslant 9 J$. Figures 4(a) and 4(b) exhibit the magnetization $M$ and its field derivative $d M / d h$ at $T=0.2 \mathrm{~J}$ over the same field range. Notice that the UUD state is very well identified by the abrupt variation of $d M / d h$, with two peaks in Fig. 4(b) representing the lower and upper critical fields. Away from the UUD state the slope of the magnetization approaches $1 /(9 J)$, which is just the uniform susceptibility of the classical antiferromagnet at zero temperature. The magnetization slope in the UUD state [see Fig. 4(a)] is visibly smaller, but clearly remains finite. This is a manifestation of the dual role played by temperature: it simultaneously selects coplanar states and thermally disorders them, eventually resulting in a paramagnetic state above a field-dependent critical temperature.

Despite being such a well studied problem, there remains a serious question about the phase diagram of the isotropic triangular lattice antiferromagnet at $\mathbf{h}=0$. As described above in Sec. III A, the large degeneracy of the model in this limit is symmetry related and reflects arbitrariness of the ordering plane in which the $120^{\circ}$ spiral forms. This makes the order parameter space isomorphic to $\mathrm{SO}(3)$, the group of rotations of a three-dimensional rigid body. ${ }^{8}$ According to the Mermin-Wagner theorem, this continuous symmetry cannot be broken at any finite temperature. Nonetheless, Monte Carlo simulations, including ours, do show a weak peak in specific heat at finite temperature, approximately $0.33 \mathrm{~J}$. It has been suggested ${ }^{8}$ that this finite-T feature in fact reflects a nontrivial phase transition associated with binding of $Z_{2}$ vortices permitted by the $\mathrm{SO}(3)$ structure. At present, our study, which aims to understand the global features of the deformed Heisenberg model at finite $T$ and $\mathbf{h}$, has nothing to add to this interesting issue, ${ }^{34-39}$ the resolution of which requires more extensive Monte Carlo simulations.

We should emphasize, however, that at finite magnetic fields, the entropically selected states break translational

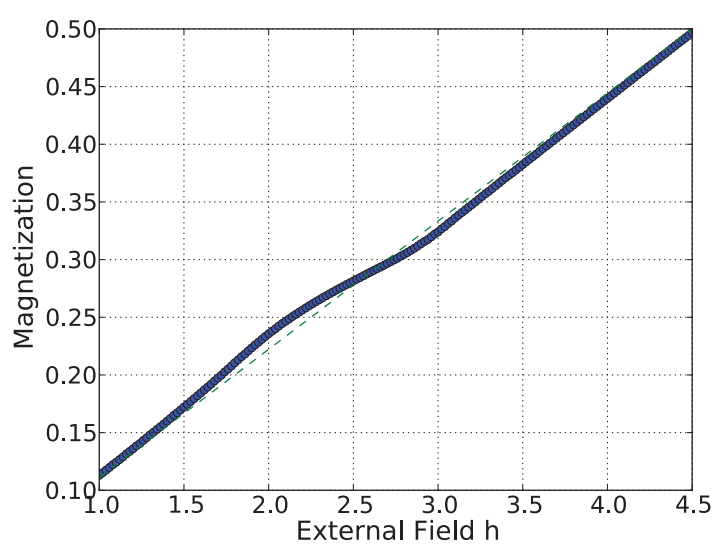

(a)

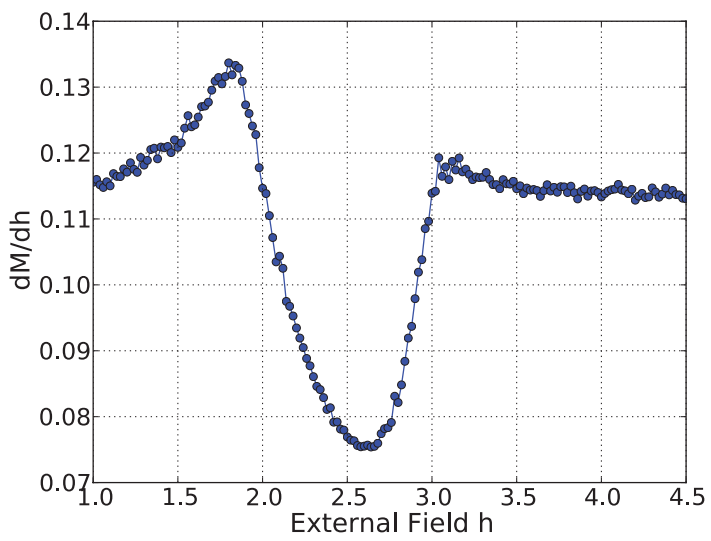

(b)

FIG. 4. (Color online) (a) Magnetization $M$ and (b) its field derivative $d M / d h$ in the vicinity of one-third magnetization. The data were obtained with Monte Carlo simulations of the classical isotropic model at temperature $T=0.2 \mathrm{~J}$. The collinear UUD state underlies the drop in magnetic susceptibility near $h=2.5 \mathrm{~J}$, visible in both (a) and (b). The dashed line in (a) shows the $T=0$ magnetization of the classical triangular antiferromagnet, $M=h /(9 J)$.

symmetry and introduce three inequivalent sublattices $A, B$, and $C$. Consequently, at any finite $\mathbf{h}$ up to saturation, there exists a finite critical temperature $T_{\mathrm{d}}$ arising from discrete symmetry breaking associated with the spontaneous selection of the sublattice ordering. In Fig. 3, this hull-shaped $T_{\mathrm{d}}(h)$ separates low-temperature ordered states from a high-temperature paramagnetic phase in which only the uniform magnetization $M$ is present.

The finite-field situation contains yet another unresolved issue, related to the $\mathrm{U}(1)$ symmetry corresponding to rotations about the magnetic field direction. The coplanar $\mathrm{Y}$ and $\mathrm{V}$ states break this symmetry by selecting an ordering direction in the plane perpendicular to the field direction. While due to dimensionality, the $\mathrm{U}(1)$ symmetry can not be truly broken at finite temperature, there exists a Kosterlitz-Thouless (KT) temperature $T_{\mathrm{KT}}$ below which the transverse magnetic order exhibits power-law correlations. In principle, one could have $T_{\mathrm{KT}} \neq T_{\mathrm{d}}$ (see Ref. 37 for a recent example of such behavior). From the available data, however, it appears that within our numerical accuracy, the two transitions coincide, $T_{\mathrm{KT}}=T_{\mathrm{d}}$. 
A related issue is that at finite temperature, the transitions from UUD to either $\mathrm{Y}$ or $\mathrm{V}$ are also of KT type, and as such are inherently broad and difficult to locate precisely in numerics. ${ }^{40} \mathrm{We}$ would like to emphasize again that our main focus is on identifying possible phases rather than studying the nature of the transitions between them. Readers interested in properties of such phase transitions in a similar context are instead referred to the recent detailed study in Ref. 40.

\section{Quantum ground states}

As first shown by Chubukov and Golosov, ${ }^{9}$ the accidental degeneracy inherent in the classical isotropic model can be lifted even at zero temperature by quantum fluctuations. Here, zero-point energy of spin waves - rather than entropyprovides the degeneracy-lifting mechanism. Quantum groundstate selection can be systematically explored using the machinery of the $1 / S$ expansion. To this end, one starts with a particular classical ground state in the degenerate manifold and defines rotated spin coordinates $\tilde{\mathbf{S}}_{A / B / C}(\mathbf{r})$ on the three sublattices such that the classical order corresponds to $\left\langle\tilde{\mathbf{S}}_{A / B / C}(\mathbf{r})\right\rangle=S \hat{\mathbf{z}}$. One then introduces Holstein-Primakoff bosons $a(\mathbf{r}), b(\mathbf{r})$, and $c(\mathbf{r})$ to express the rotated spin operators to leading order in $1 / S$ as

$$
\begin{gathered}
\tilde{S}_{A}^{x}(\mathbf{r})=\sqrt{\frac{S}{2}}\left[a(\mathbf{r})+a^{\dagger}(\mathbf{r})\right], \\
\tilde{S}_{A}^{y}(\mathbf{r})=i \sqrt{\frac{S}{2}}\left[a(\mathbf{r})-a^{\dagger}(\mathbf{r})\right], \\
\tilde{S}_{A}^{z}(\mathbf{r})=S-a^{\dagger}(\mathbf{r}) a(\mathbf{r}),
\end{gathered}
$$

and similarly for $\tilde{\mathbf{S}}_{B / C}(\mathbf{r})$. Retaining only the leading-order terms in $1 / S$ generates a Hamiltonian of the form $H=E_{0}+$ $H_{2}$, where $E_{0}$ is the classical ground-state energy and $H_{2}$ is quadratic in the three boson fields.

Although at a given magnetic field $h, E_{0}$ is identical for all allowed classical ground states satisfying Eq. (7), the spin-wave Hamiltonian $\mathrm{H}_{2}$ does depend on the particular ordered configuration around which one is expanding, via the dependence of the spin-wave dispersion $\omega_{k}$ on the angles $\theta_{A / B / C}$ and $\phi_{A / B / C}$. The zero-point energy for spin-waves, $\left\langle H_{2}\right\rangle=(1 / 2) \sum_{k} \omega_{k}$, therefore yields a $1 / S$ correction to the energy that lifts the classical degeneracy. See, for example, Ref. 41 for an earlier application of this strategy to a related problem. We note that this analysis can be carried out rather efficiently by parametrizing the original spin operators via Eq. (6) and deriving the general form of $H_{2}$ as outlined above. The constraints in Eqs. (7) allow one to express $\phi_{B}$, $\phi_{C}$, and $\theta_{C}$ in terms of $\phi_{A}, \theta_{A}$, and $\theta_{B}$; due to the U(1) spin symmetry that is present when $\mathbf{h} \neq 0$, one can arbitrarily set $\phi_{A}=0$. Consequently, $H_{2}$ can be expressed in terms of only two angles, $\theta_{A / B}$. Diagonalizing $H_{2}$ for a mesh of $\theta_{A}$ and $\theta_{B}$ then allows one to compute the zero-point energies for the classical ground-state manifold to deduce which states quantum fluctuations favor. Although the selection for the isotropic model is already well known, we will use this scheme later on to explore the competition between quantum fluctuations and DM interactions.

It is important to emphasize a subtle point in the procedure outlined above. At order $1 / S$ quantum fluctuations not only split the degeneracy through zero-point motion, but also renormalize the spin structure in the classical ground states. Thus to order $1 / S$, the spin angles should read $\theta_{A}=\theta_{A}^{0}+\delta \theta_{A}$, and similarly for other angles, where $\theta_{A}^{0}$ corresponds to a classical ground state and $\delta \theta_{A}$ is a $1 / S$ quantum correction (which we do not calculate here). When evaluating the zeropoint energies $\left\langle H_{2}\right\rangle$ to order $S$, clearly one can safely neglect such quantum corrections to the spin states. One may worry, however, that since the classical ground-state energy $E_{0}$ is proportional to $S^{2}$, evaluating $E_{0}$ with the renormalized spin angles may produce an order- $S$ contribution to the energy that is comparable to the leading zero-point energy. This is certainly not the case-because the classical ground states minimize $E_{0}$, the shifts $\delta \theta_{A}$ appear only at second order and therefore contribute to the energy only at order $S^{0}$; see, e.g., the discussion in Ref. 42. Thus while renormalization of the spin structure is crucial for capturing certain physical quantities such as quantum corrections to the magnetization curve as a function of field, this effect is indeed unimportant for our main purpose: evaluating the ground-state energies to leading order in $1 / S$.

As in the case of thermal fluctuations, quantum fluctuations disfavor noncoplanarity and prefer collinear order, leading to $\mathrm{Y}$ states below $1 / 3$ magnetization $\left(0<h<h_{\text {sat }} / 3\right)$, UUD ordering at $1 / 3$ magnetization $\left(h=h_{\text {sat }} / 3\right)$, and V states at larger magnetization ( $h_{\text {sat }} / 3<h<h_{\text {sat }}$ ) up to saturation. ${ }^{9}$ While it is well known that the UUD phase realizes a magnetization plateau also in the quantum problem, ${ }^{9}$ we emphasize that this fact arises from higher-order quantum corrections such as the $S^{0}$ effect discussed above. One can recover this feature by incorporating $1 / S$ quantum renormalization of the equilibrium angles for the $\mathrm{Y}$ and $\mathrm{V}$ states, which broadens the UUD state into a plateau. ${ }^{9}$ This result can be also be seen in the following complementary way. Since the UUD state is collinear, it does not break any continuous symmetry, and therefore exhibits only gapped spin excitations when one renormalizes the spin-wave spectrum to leading order in $1 / S$ (note that such a renormalization shifts the ground-state energy only at order $S^{0}$ ). Thus a finite change in the magnetic field is required to destabilize this phase.

To establish intuition that will prove beneficial in subsequent sections, it is useful to now ask how robust the quantum selection of Y/UUD/V order is at different magnetic fields. One can quantify this by computing the first $1 / S$ quantum correction to the energy, i.e., evaluating $\left\langle H_{2}\right\rangle$, for the Y/UUD/V states and comparing it with the corresponding correction for other classical ground states that quantum fluctuations disfavor. The size of these energy differences $\Delta E=(1 / 2) \sum_{k}\left[\omega_{k}^{\text {other }}-\omega_{k}^{\mathrm{Y} / \mathrm{UUD} / \mathrm{V}}\right]$ provide one with a rough guide for how robust the quantum ground-state selection is to the inclusion of other perturbations, such as DM interactions. Figure 5 presents the energy splittings $\Delta E$ obtained for the umbrella (solid line) and inverted-Y states (dashed line) as a function of $h$. To produce the data shown, we have extrapolated large- $S$ corrections to $S=1 / 2$. It is seen that Y/UUD/V order is preferred over umbrella and inverted-Y states for all values of the field. It is also seen that being coplanar, the inverted-Y state has lower energy than the umbrella one. Although only two states, umbrella and inverted-Y, are considered, the figure 


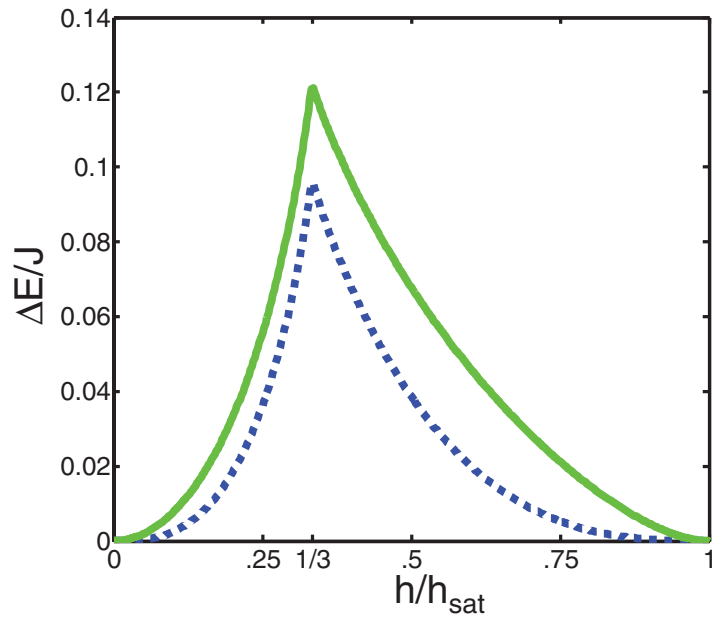

FIG. 5. (Color online) Leading $1 / S$ quantum energy splittings between the Y/UUD/V states and the inverted-Y (dashed line) and umbrella states (solid line). These curves illustrate an important trend-quantum fluctuations are most effective at splitting the accidental degeneracy near $1 / 3$ magnetization where low-energy collinear states are available.

clearly illustrates a general trend that recurs throughout the paper-quantum fluctuations are most effective at selecting ground states in the vicinity of $1 / 3$ magnetization where low-energy collinear spin configurations are available.

\section{E. Biquadratic approximation of quantum fluctuations (zero-point motion)}

There is an alternative, albeit less rigorous, way to account for the influence of quantum fluctuations, within a purely classical spin Hamiltonian. This approach is based on the observation that coplanar and especially collinear spin stateswhich quantum fluctuations tend to favor most strongly - can be stabilized classically upon adding a suitable biquadratic spin coupling to the Hamiltonian. ${ }^{9}$ This biquadratic interaction represents an effective Hamiltonian that is generated by quantum and/or thermal fluctuations. ${ }^{43}$ We have incorporated such a biquadratic spin interaction and optimized its (fielddependent) coupling constant such that we reproduce the leading $1 / S$ energy differences between several important states in the classical ground-state manifold. In this way, we find that quantum zero-point energy can be semiquantitatively described by the following classical biquadratic Hamiltonian with negative coupling constant:

$$
\begin{gathered}
\delta H_{\mathrm{bi}}=-\Delta(h) \sum_{\left\langle\mathbf{r r}^{\prime}\right\rangle}\left(\mathbf{S}_{\mathbf{r}} \cdot \mathbf{S}_{\mathbf{r}^{\prime}}\right)^{2}, \\
\Delta(h)=\frac{0.0268}{S}\left(1-0.03 h \sqrt{h_{\mathrm{sat}}-h}\right) .
\end{gathered}
$$

Note that spins $\mathbf{S}_{\mathbf{r}}$ appearing in $\delta H_{\mathrm{bi}}$ are classical unit vectors and that all dependence on the magnitude of the microscopic spin $S$ is contained in the field-dependent coupling constant $\Delta(h)$. Figure 6 compares the energy differences between various classical ground states specified in the caption using the leading $1 / S$ results (solid lines) and the biquadratic classical approximation (dashed lines), showing the quite good quantitative agreement between the two approaches.

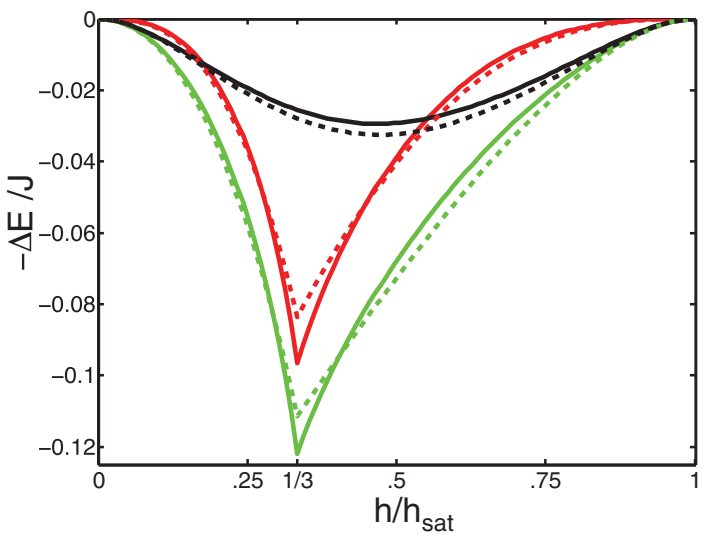

FIG. 6. (Color online) Energy differences between various states using the leading $1 / S$ result from spin-wave theory (solid lines) and the biquadratic classical approximation (dashed lines). Green lines represent the difference between $\mathrm{Y} / \mathrm{UUD} / \mathrm{V}$ and inverted-Y energies, red between $\mathrm{Y} / \mathrm{UUD} / \mathrm{V}$ and umbrella, and black between inverted-Y and umbrella.

The full benefit of approximating quantum zero-point energy by the classical biquadratic interaction will become clear when we address the effects of spatial anisotropy $J^{\prime} \neq J$ in Secs. VIB and VIIB. The classical energy gain due to spatial anisotropy is quadratic in the difference $J-J^{\prime}$. Such second-order corrections are not in general easy to account for analytically, making it difficult to consistently compare the anisotropy-induced energy splittings with those arising from quantum effects and DM interactions. Using the biquadratic interaction in Eq. (13), however, allows us to circumvent this problem and find the result of the competition between the different perturbations by numerically studying, via standard Monte Carlo techniques, the classical system described by $H_{0}+H_{\mathrm{DM}}+\delta H_{\mathrm{bi}}$. Later, we will refer to ground states obtained within such a classical model with biquadratic spin couplings as "pseudoquantum ground states" to emphasize that despite corresponding to classical spins, such states are expected to semiquantitatively reflect the influence of quantum fluctuations.

As a warm-up exercise of such a strategy, we first simulate a simpler "isotropic-plus-biquadratic" Hamiltonian $H_{\text {iso+bi }}=$ $H_{0}+\delta H_{\text {bi }}$. Figure 7 shows $d M / d h$ and the coplanarity $K$ for this model at a low temperature of $T=0.027 \mathrm{~J}$. This low temperature was reached via simulated annealing to avoid becoming trapped in local (but not global) free-energy minima. Specifically, Monte Carlo simulations were performed on a $24 \times 24$ system starting at $T=0.4 \mathrm{~J}$, then cooling down in small $\Delta T=0.009 \mathrm{~J}$ steps. At each temperature, the system was equilibrated with 35000 Monte Carlo steps. Measurements were taken during the last 30000 steps.

The pronounced minimum of $d M / d h$ visible in Fig. 7 over the field interval $2.2 J \lesssim h \lesssim 3.2 J$ identifies this region with the collinear UUD state. The coplanar nature of the adjacent phases is evident from the plot of $K$ versus $h$ in the same figure. Note that despite the very low temperature considered, a substantial UUD plateau remains whose width greatly exceeds that of the purely classical model without biquadratic interactions; see Fig. 3 for comparison. The width of the plateau is in fact proportional to $\Delta(h)$ as discussed in Ref. 9; 


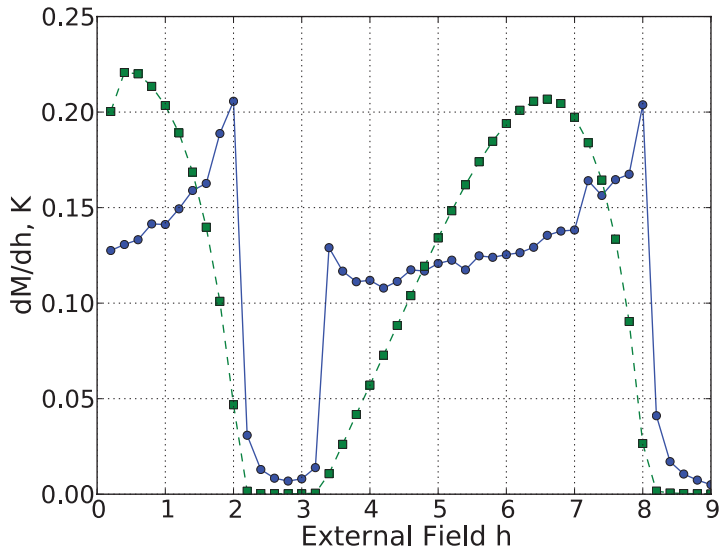

FIG. 7. (Color online) $d M / d h$ (blue circles) and coplanarity $K$ (green squares), defined in Eq. (11), for the classical "isotropic-plusbiquadratic" model at $T=0.027 \mathrm{~J}$, obtained by annealing from high temperature.

quantum fluctuations, modeled here by $\delta H_{\mathrm{bi}}$, produce a stable UUD magnetization plateau over a finite field interval even at $T=0$. We note that the usual spin-wave approach predicts a plateau over a field interval of width $\Delta h \approx 1.8 \mathrm{~J} /(2 S)$, which is somewhat larger than the plateau width obtained in our isotropic-plus-biquadratic simulations.

\section{SPATIALLY ISOTROPIC MODEL WITH DZYALOSHINSKII-MORIYA INTERACTIONS: $J=J^{\prime}, D \neq 0$}

In this section, we will explore how DM coupling affects the classical and quantum phase diagrams discussed above. One complication that arises here is that once we invoke DM interactions, the magnetic field direction is no longer arbitrary as it was in the previous section. Recall that the DM vector points along the $z$ axis. We will begin in Sec. IV A with the case where the field is applied along the $z$ direction, perpendicular to the triangular lattice plane. This field orientation is simplest to analyze since the Hamiltonian including $H_{\mathrm{DM}}$ then preserves global U(1) spin symmetry about the $z$ axis. In-plane field orientations are studied in Sec. IV B.

We should also emphasize that in any field orientation, DM coupling breaks $\pi$ /3-rotation symmetry since $H_{\mathrm{DM}}$ in Eq. (2) only couples spins on neighboring chains of the lattice. Thus the model with only DM coupling (and no spatial anisotropy) is clearly fine tuned. Nevertheless, by examining the effects of DM alone, we will gain valuable intuition that will be useful when we include the additional complication of spatial anisotropy in Sec. VII.

\section{A. Parallel orientation: $\mathbf{D} \| \mathbf{h}=\boldsymbol{h} \hat{\mathbf{z}}$ \\ 1. Classical ground states}

We will first explore how DM interactions lift the accidental degeneracy of the classical isotropic model using first-order perturbation theory. At this order, one simply needs to evaluate the DM energy using the unperturbed form of the classical ground states found in the previous section.
Using the parametrization for the ground states (6) and the constraints (7), we obtain

$$
\begin{aligned}
\left\langle H_{\mathrm{DM}}\right\rangle= & 2 \sigma N D\left\{-\sin ^{2} \theta_{A} \sin ^{2} \theta_{B}+\frac{1}{324 J^{4}}\left[h^{2}+9 J^{2}\right.\right. \\
& \left.\left.-6 J h\left(\cos \theta_{A}+\cos \theta_{B}\right)+18 J^{2} \cos \theta_{A} \cos \theta_{B}\right]^{2}\right\}^{1 / 2},
\end{aligned}
$$

where $\sigma=\operatorname{sign}\left[\sin \left(\phi_{A}-\phi_{B}\right)\right]$ determines the chirality of the spins in the $(x, y)$ plane. The DM energy above is minimized when $\cos \theta_{A}=\cos \theta_{B}=\frac{h}{9 J}$ and $\sigma=-1$. One can readily see using Eq. (7) that this corresponds to umbrella states of Fig. 2(f) for all fields (with a specific chirality because $D \neq 0$ breaks inversion symmetry). This outcome is quite natural given that DM coupling at $\mathbf{h}=0$ favors coplanar spiral order with spins pointing in the triangular lattice plane; the field simply cants the spiral out of the plane, producing an umbrella pattern. Note that this is markedly different from the coplanar and collinear configurations favored by thermal and quantum fluctuations. This leads to an interesting competition once one incorporates these additional ingredients, as we now address.

\section{Influence of thermal fluctuations}

One can deduce the qualitative outcome of the competition between DM interactions and thermal fluctuations at finite temperature using the following general argument. In the previous section, we discussed that while at $T=0$ the isotropic model exhibits many classically degenerate ground states, at finite temperature entropy lifts this degeneracy in favor of Y/UUD/V states. Moreover, this selection is strongest at intermediate temperatures where thermal fluctuations provide a large free-energy splitting but are not so severe as to destroy ordering altogether. (This can be seen most clearly through the width of the UUD plateau in Fig. 3, which reaches at maximum at around $T=0.35 \mathrm{~J}$ before entering the paramagnetic phase at slightly higher $T$.)

Now suppose one slowly turns on DM interactions. At zero temperature, any finite DM coupling will immediately select umbrella ordering since the entropic splittings vanish as $T \rightarrow 0$. By continuity, umbrella states must persist over a finite temperature window that depends on both field and the DM coupling strength. Similarly, at intermediate temperatures, the entropically favored Y/UUD/V states must also by continuity survive some amount of DM coupling. This leads to multistage ordering as a function of temperature. Consider for illustration the interesting example of one-third magnetization with weak DM coupling. As temperature increases from zero, the system will begin in an umbrella state, transition into an UUD plateau at intermediate temperatures, and eventually give way to a paramagnet at high temperatures.

The qualitative picture that emerges is that as one increases DM coupling from zero, DM interactions eat away at the entropically-stabilized regions from the low-temperature side, eventually removing the Y/UUD/V phases entirely in favor of umbrella order at sufficiently large coupling. Obtaining a more quantitative understanding of this competition requires extensive Monte Carlo simulations, which we will not explore here. We will instead perform such a study in the more 
physically relevant case with spatial anisotropy in Sec. VI A, where very similar physics arises. It should also be kept in mind that in this subsection we neglected quantum effects entirely. Below, we incorporate quantum fluctuations and show that they lead to a substantially richer zero-temperature phase diagram than that of the purely classical model.

\section{Quantum ground states}

To incorporate DM interactions and quantum fluctuations at $T=0$, we treat $1 / S$ and $D / J$ as expansion parameters of the same order of magnitude. The first-order corrections to the classical ground-state energies can then be obtained simply by adding the $1 / S$ spin-wave contribution discussed in Sec. III D to the classical DM energy in Eq. (14). [For example, the effect of quantum fluctuations on the DM energy produces a higher-order correction $\sim(D / J)(1 / S)$.] By evaluating the perturbed energies for a dense subset of classical ground states as described in Sec. III D, one can determine the spin order selected when both competing effects are present.

Carrying out this procedure with $D / J=0.05$ and $S=1 / 2$, we have found that the umbrella phase is the quantum ground state for sufficiently small magnetization $\left(h / h_{\text {sat }} \lesssim\right.$ $0.3)$ and, also, sufficiently near saturation $\left(h / h_{\text {sat }} \gtrsim 0.94\right)$; see Fig. 8(a), which summarizes our numerics for this case. In between, however, a different ground state emerges, which reflects a compromise between DM interactions and quantum fluctuations - the distorted V phase of Fig. 2(d). This state resembles the $\mathrm{Y}$ and $\mathrm{V}$ orders favored by quantum fluctuations, but is distorted in a noncoplanar fashion to gain DM energy. Note that the distortion of the spins away from perfect $Y$ or $\mathrm{V}$ order is not unexpected: these states as well as UUD exhibit rotation symmetry, which is explicitly broken by the DM interaction in Eq. (2) (recall the discussion at the end of Sec. III B). The analytical structure of the distorted-V state is

(a)

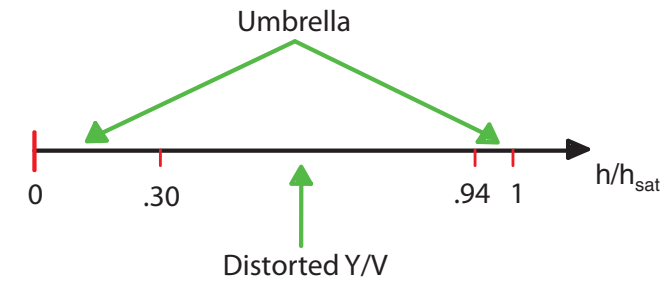

(b)

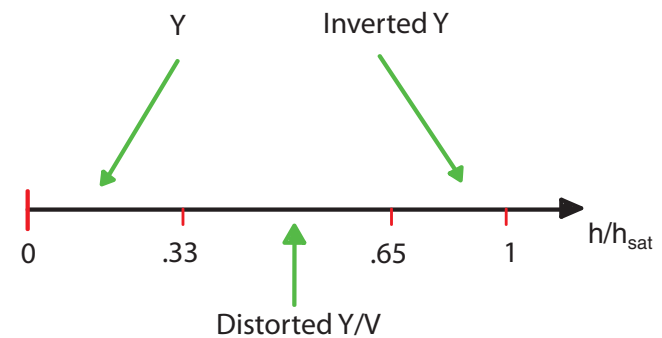

FIG. 8. (Color online) Phase diagram of the quantum Heisenberg model with DM interactions, as a function of magnetic field $h$. The magnetic field orients along the DM axis, $\mathbf{D} \| \mathbf{h}$, in (a) and orthogonal to the DM axis, $\mathbf{D} \perp \mathbf{h}$, in (b). easy to obtain in the high-magnetic-field limit as described in Sec. V B.

\section{B. Orthogonal orientation: $\mathrm{D} \perp \mathrm{h}=h \hat{\mathbf{x}}$}

\section{Classical ground states}

We will now extend the analysis of the previous section to the case where the magnetic field is applied in the plane of the triangular layers, along the $x$ direction for concreteness. This field orientation is complicated by the fact that the quantum Hamiltonian including DM coupling now lacks U(1) spin symmetry; instead, the Hamiltonian is symmetric only under discrete spin rotations $S_{\mathbf{r}}^{x} \rightarrow S_{-\mathbf{r}}^{x}$ and $S_{\mathbf{r}}^{y, z} \rightarrow-S_{-\mathbf{r}}^{y, z}$. As above, we start by deducing the order favored by DM coupling in this field orientation using first-order perturbation theory. Using Eq. (6) to parametrize the classical ground states along with the constraints in Eq. (7), the first-order correction to the energy arising from DM coupling can be written:

$$
\begin{aligned}
\left\langle H_{\mathrm{DM}}\right\rangle= & -\frac{2 D N}{9 J}\left[h\left(\sin \theta_{B} \sin \phi_{B}-\sin \theta_{A} \sin \phi_{A}\right)\right. \\
& \left.+9 J \sin \theta_{A} \sin \theta_{B} \sin \left(\phi_{A}-\phi_{B}\right)\right],
\end{aligned}
$$

where the angles are subject to the additional constraint

$$
\begin{aligned}
\frac{1}{2}[ & \left.+\left(\frac{h}{3 J}\right)^{2}\right] \\
= & \frac{h}{3 J}\left(\sin \theta_{A} \cos \phi_{A}+\sin \theta_{B} \cos \phi_{B}\right) \\
& \quad-\sin \theta_{A} \sin \theta_{B} \cos \left(\phi_{A}-\phi_{B}\right)-\cos \theta_{A} \cos \theta_{B} .
\end{aligned}
$$

One can use Eq. (16) to eliminate $\theta_{B}$ from Eq. (15) and then minimize the DM energy over the remaining angles $\theta_{A}, \phi_{A}$, and $\phi_{B}$. In this way, we find that DM coupling selects coplanar inverted-Y states of Fig. 2(e), with

$$
\begin{gathered}
\theta_{A / B / C}=\pi / 2, \\
\phi_{A}=-\phi_{B}=\cos ^{-1}\left[\frac{1}{2}\left(\frac{h}{3 J}-1\right)\right], \\
\phi_{C}=0 .
\end{gathered}
$$

The emergence of planar ground states for this field orientation is quite natural, again because DM interactions favor spins orienting within the triangular lattice plane. Among the various planar arrangements, inverted-Y states most effectively gain DM energy by keeping neighboring spins as far from parallel as possible (within the constraints set by the ground-state manifold). In contrast, the UUD and V states favored by quantum fluctuations yield a vanishing first-order DM energy because in both configurations spins on two sublattices are parallel. We note that the favorability of inverted-Y over $Y$ states at low fields is less obvious; the latter also gains DM energy, though to a lesser extent than the former.

Just as in the $\mathbf{h}=h \hat{\mathbf{z}}$ field orientation, incorporating thermal or quantum fluctuations therefore leads to a delicate competition with DM interactions. The discussion from Sec. IV A 2 regarding entropic effects applies in this field orientation as well. Perhaps the most important qualitative distinction with the $\mathbf{h}=h \hat{\mathbf{z}}$ field orientation is the additional loss of U(1) symmetry here due to the orthogonality between $\mathbf{h}$ and $\mathbf{D}$ vectors. This implies the demise of the 1/3-magnetization 
plateau since the UUD and Y states then no longer constitute distinct phases separated by a spontaneous U(1) symmetry breaking. Section VII B discusses this effect in greater detail; see Fig. 15 for an illustration of the rounding-off of the magnetization plateau by DM interactions.

In the following subsection, we will resolve the outcome of the competition between quantum fluctuations and DM interactions at zero temperature, which is qualitatively different from the previously considered $\mathbf{h}=h \hat{\mathbf{z}}$ case.

\section{Quantum ground states}

The quantum zero-temperature phase diagram in this field orientation is obtained as described in Sec. IV A 3, again treating quantum corrections and DM coupling to first order with $D / J=0.05$ and $S$ extrapolated to $1 / 2$. Figure $8(\mathrm{~b})$ depicts our results. Quantum effects and DM coupling compete in a subtle manner for this field orientation. The former dominates at low fields, where Y states prevail, while the latter dominates at high fields where inverted-Y states appear. Distorted-V order, which reflects a nontrivial compromise between the two competing effects, appears at intermediate magnetization, $1 / 3 \lesssim h / h_{\text {sat }} \lesssim 0.65$. Note that all states are either perfectly planar or very nearly so here, which again is rather natural. Also notable is the absence of the collinear UUD state in the quantum phase diagram. As argued above, this is caused by the loss of U(1) spin symmetry in the $\mathbf{D} \perp \mathbf{h}$ geometry.

Observe that the quantum phase diagrams predicted for the two field orientations, $\mathbf{h} \propto \hat{\mathbf{z}}$ and $\mathbf{h} \propto \hat{\mathbf{x}}$, are very different. At either low or high fields, this implies that at least one phase transition separating the umbrella and Y/inverted-Y states ought to appear as one rotates the field from the $z$ to the $x$ direction. Recent experiments ${ }^{28}$ do indeed find a very complicated evolution of the phase diagram as the field rotates in this plane, featuring several intervening phase transitions. Generalizing the results of this paper to explore this crossover would be an interesting future research direction.

\section{BOSE-EINSTEIN CONDENSATION ANALYSIS NEAR SATURATION}

We will now shift our focus to phases realized at high magnetic fields near saturation, which can be elegantly described via Bose-Einstein condensation (BEC) of magnon excitations above the fully polarized (saturated) state. ${ }^{4-48}$ In this way, magnetically ordered phases just below saturation ( $\left.h=h_{\text {sat }}-\delta h\right)$ can be understood as a BEC instability of the ground state just above saturation $\left(h=h_{\text {sat }}+\delta h\right)$. This limit has the advantage of allowing one to incorporate all of the competing effects of interest-quantum fluctuations, DM coupling, and spatial anisotropy-in a single controlled and coherent framework.

\section{A. Preliminaries: $J=J^{\prime}, D=0$}

At $h>h_{\text {sat }}$, the ground state of the Heisenberg Hamiltonian is known exactly: it is given by the fully polarized eigenstate of the Hamiltonian in which all spins point up. The excitations are magnons, i.e., plane-wave states of overturned spins. These are conveniently described by Holstein-Primakoff bosons, similar to Eq. (12). Since all spins point in the same direction, it suffices to consider only a single boson species here; thus we write

$$
S^{z}(\mathbf{r})=S-a^{\dagger}(\mathbf{r}) a(\mathbf{r}), \quad S^{+}(\mathbf{r})=\sqrt{2 S} a(\mathbf{r}),
$$

for all $\mathbf{r}$. The isotropic Heisenberg Hamiltonian without DM coupling then reads, neglecting $1 / S$ and smaller contributions,

$$
\begin{gathered}
H=H_{0}+V \\
H_{0}=\sum_{\mathbf{k}} S[J(\mathbf{k})-J(\mathbf{Q})-\mu] a_{\mathbf{k}}^{\dagger} a_{\mathbf{k}} \\
V=\frac{1}{4 N} \sum_{\mathbf{k}, \mathbf{k}^{\prime}, \mathbf{q}}\left[2 J(\mathbf{q})-J(\mathbf{k})-J\left(\mathbf{k}^{\prime}-\mathbf{q}\right)\right] a_{\mathbf{k}+\mathbf{q}}^{\dagger} a_{\mathbf{k}^{\prime}-\mathbf{q}}^{\dagger} a_{\mathbf{k}^{\prime}} a_{\mathbf{k}} .
\end{gathered}
$$

Here,

$$
\begin{aligned}
J(\mathbf{k}) & =\sum_{j=1}^{3} J_{\mathbf{r}, \mathbf{r}+\boldsymbol{\delta}_{j}} \cos \left(\mathbf{k} \cdot \boldsymbol{\delta}_{j}\right) \\
& =J\left[\cos \left(k_{x}\right)+2 \cos \left(k_{x} / 2\right) \cos \left(\sqrt{3} k_{y} / 2\right)\right]
\end{aligned}
$$

is the Fourier transform of the exchange interaction, and

$$
\mu=\left(h_{\text {sat }}-h\right) / S
$$

is the chemical potential, which controls the state of the bosonic system. For $h>h_{\text {sat }}$, the chemical potential is negative and the ground state is the boson vacuum $|0\rangle$ satisfying $a_{\mathbf{k}}|0\rangle=0$ for all $\mathbf{k}$. The wave vector $\mathbf{Q}$ in Eq. (19) corresponds to the minimum of the magnon energy and follows from $J(\mathbf{Q})=\min _{\mathbf{k}}[J(\mathbf{k})]$. A peculiarity of the triangular antiferromagnet, ${ }^{45}$ and indeed of many other frustrated spin systems, ${ }^{48}$ is the fact that there are two distinct wave vectors minimizing $J(\mathbf{k})$. For the isotropic two-dimensional triangular antiferromagnet, these are $\pm \mathbf{Q}=( \pm 4 \pi / 3,0)$. Hence, at $h=$ $h_{\text {sat }}$ (or equivalently $\mu=0$ ), the magnon dispersion touches zero simultaneously at $\pm \mathbf{Q}$. One then needs to understand whether it is energetically favorable to condense magnons at one or both of these wave vectors, i.e., form a single- $Q$ or double- $Q$ condensate.

The analysis proceeds by parametrizing the magnon modes as follows:

$$
a_{\mathbf{k}}=\sqrt{N} \psi_{+Q} \delta_{\mathbf{k}, \mathbf{Q}}+\sqrt{N} \psi_{-Q} \delta_{\mathbf{k},-\mathbf{Q}}+\tilde{a}_{\mathbf{k}} .
$$

Here, $\tilde{a}_{\mathbf{k}}$ describes noncondensed magnons with $\mathbf{k} \neq \pm \mathbf{Q}$. The leading contribution to the energy (per site) is obtained by neglecting the noncondensed particles altogether. Written in terms of condensate densities $\rho_{1,2}=\left|\psi_{ \pm Q}\right|^{2}$, the energy reads

$$
\frac{E}{N}=-S \mu\left(\rho_{1}+\rho_{2}\right)+\frac{1}{2} \Gamma_{1}\left(\rho_{1}^{2}+\rho_{2}^{2}\right)+\Gamma_{2} \rho_{1} \rho_{2} .
$$

The coefficients $\Gamma_{1,2}$ will be defined momentarily below, but first, it is useful to discuss the important physics described by this simple equation in some generality. When $\mu<0$, the energy is minimized by a vacuum state with no condensate, $\rho_{1,2}=0$. Increasing $\mu$ (by lowering $h$ ) to positive values leads to a condensed state. A double- $Q$ condensate emerges for $\Gamma_{1}>$ $\Gamma_{2}$, when $\rho_{1}=\rho_{2}=S \mu /\left(\Gamma_{1}+\Gamma_{2}\right)$. The physical meaning of this is found by expressing the spin operators in terms of 
boson condensates. Using $\psi_{ \pm Q}=\sqrt{\rho_{1}} e^{i \theta_{1,2}}$ and writing $\theta_{1,2}=$ $\theta_{+} \pm \theta_{-}$, we find

$$
\begin{gathered}
S_{\mathbf{r}}^{+}=2 S \sqrt{\frac{2 \mu}{\Gamma_{1}+\Gamma_{2}}} e^{i \theta_{+}} \cos \left[\mathbf{Q} \cdot \mathbf{r}+\theta_{-}\right], \\
S_{\mathbf{r}}^{z}=S-\frac{8 \mu S}{\Gamma_{1}+\Gamma_{2}} \cos ^{2}\left[\mathbf{Q} \cdot \mathbf{r}+\theta_{-}\right] .
\end{gathered}
$$

Hence, the double- $Q$ condensate describes a coplanar magnetically ordered state. Observe that the $S^{z}$ spin component is modulated with wave vector $2 \mathbf{Q}$. The coplanar state described by Eq. (24) thus represents a supersolid phase of the magnet. A single- $Q$ condensate arises when $\Gamma_{1}<\Gamma_{2}$; in this case, $\rho_{1}=S \mu / \Gamma_{1}$ and $\rho_{2}=0$ or vice versa. This state corresponds to a conventional umbrella (or cone) magnetic structure:

$$
\begin{aligned}
S_{\mathbf{r}}^{+} & =S \sqrt{\frac{2 \mu}{\Gamma_{1}}} \exp \left[i \mathbf{Q} \cdot \mathbf{r}+i \theta_{1}\right], \\
S_{\mathbf{r}}^{z} & =S-\frac{\mu S}{\Gamma_{1}} .
\end{aligned}
$$

The classical (order $1 / S^{0}$ ) expression for $\Gamma_{1,2}$ is given by

$$
\Gamma_{1}=J(0)-J(\mathbf{Q}), \Gamma_{2}=J(0)-2 J(\mathbf{Q})+J(2 \mathbf{Q}) .
$$

The accidental degeneracy of the classical isotropic triangular antiferromagnet discussed in Sec. III A manifests itself via the relation $\Gamma_{1}-\Gamma_{2}=J(\mathbf{Q})-J(2 \mathbf{Q})=0$ for $\mathbf{Q}=(4 \pi / 3,0)$. Thus the coplanar and cone states are degenerate classically. At first order in $1 / S$, one finds

$$
\Gamma_{1}>\Gamma_{2}
$$

so that quantum fluctuations select coplanar state, consistent with our earlier spin-wave analysis. The corresponding calculation is sketched in Refs. 45,48 and will not be discussed here for brevity. Instead, here, we take Eq. (27) as given and ask how the addition of weak DM interaction in Eq. (2) as well as spatial anisotropy influences the delicate balance between coplanar and umbrella states at high magnetic fields.

\section{B. Spatially isotropic model with Dzyaloshinskii-Moriya interactions: $J=J^{\prime}, D \neq 0, \mathrm{~h}=h \hat{\mathbf{z}}$}

In the geometry with $\mathbf{h}=h \hat{\mathbf{z}}$, the DM Hamiltonian (2) reduces to a simple quadratic form of magnon operators,

$$
\begin{aligned}
H_{\mathrm{DM}} & =\frac{i D}{2} \sum_{\mathbf{r}, j=1,3}\left(S_{\mathbf{r}}^{-} S_{\mathbf{r}+\boldsymbol{\delta}_{j}}^{+}-\text {H.c. }\right) \\
& =i D S \sum_{\mathbf{r}, j=1,3}\left(a_{\mathbf{r}}^{\dagger} a_{\mathbf{r}+\boldsymbol{\delta}_{j}}-\text { H.c. }\right) \\
& =-2 N D S\left[\sin \left(\mathbf{Q} \cdot \boldsymbol{\delta}_{1}\right)+\sin \left(\mathbf{Q} \cdot \boldsymbol{\delta}_{3}\right)\right]\left(\rho_{1}-\rho_{2}\right) .
\end{aligned}
$$

As expected physically, DM coupling breaks the symmetry between $\pm \mathbf{Q}$ points, and lowers the energy of the $\psi_{+Q}$ condensate (for positive $D>0$ ). The ground state follows from minimizing

$$
\begin{aligned}
\frac{E}{N}= & -S \mu\left(\rho_{1}+\rho_{2}\right)-S g\left(\rho_{1}-\rho_{2}\right)+ \\
& +\frac{1}{2} \Gamma_{1}\left(\rho_{1}^{2}+\rho_{2}^{2}\right)+\Gamma_{2} \rho_{1} \rho_{2},
\end{aligned}
$$

where we introduced $g=2 \sqrt{3} D>0$. Following Ref. 49, we parametrize the densities as

$$
\begin{aligned}
& \rho_{1}=\rho \cos ^{2} \phi, \\
& \rho_{2}=\rho \sin ^{2} \phi,
\end{aligned}
$$

and minimize the energy with respect to $\phi$ and $\rho$.

Three possible solutions exist:

$$
\begin{gathered}
\text { (i) } \phi=0, \quad \rho=\frac{S(\mu+g)}{\Gamma_{1}}, \quad \frac{E}{N}=-\frac{S^{2}(\mu+g)^{2}}{2 \Gamma_{1}}, \\
\text { (ii) } \phi=\frac{\pi}{2}, \quad \rho=\frac{S(\mu-g)}{\Gamma_{1}}, \quad \frac{E}{N}=-\frac{S^{2}(\mu-g)^{2}}{2 \Gamma_{1}}, \\
\text { (iii) } \cos ^{2} \phi=\frac{1}{2}\left[1+\frac{g\left(\Gamma_{1}+\Gamma_{2}\right)}{\mu\left(\Gamma_{1}-\Gamma_{2}\right)}\right], \quad \rho=\frac{2 \mu S}{\Gamma_{1}+\Gamma_{2}} \\
\frac{E}{N}=-\frac{\mu^{2} S^{2}}{\Gamma_{1}+\Gamma_{2}}-\frac{g^{2} S^{2}}{\Gamma_{1}-\Gamma_{2}} .
\end{gathered}
$$

Solutions (i) and (ii) describe umbrella states formed from a single- $Q$ condensate. For positive $g$, the umbrella corresponding to (i) with $\rho_{1} \neq 0, \rho_{2}=0$ has lower energy. The "mixed" solution (iii), which only exists when $-1 \leqslant g\left(\Gamma_{1}+\right.$ $\left.\Gamma_{2}\right) / \mu\left(\Gamma_{1}-\Gamma_{2}\right) \leqslant 1$, yields the lowest energy, provided that Eq. (27) is satisfied. This solution represents the distorted$\mathrm{V}$ state of Fig. 2(d), discussed in Sec. IV A 3 above. The condensates here satisfy

$$
\begin{aligned}
& \left|\psi_{+Q}\right|^{2}=\frac{\mu S}{\Gamma_{1}+\Gamma_{2}}+\frac{g S}{\Gamma_{1}-\Gamma_{2}}, \\
& \left|\psi_{-Q}\right|^{2}=\frac{\mu S}{\Gamma_{1}+\Gamma_{2}}-\frac{g S}{\Gamma_{1}-\Gamma_{2}},
\end{aligned}
$$

and, as a result, the spin structure is noncoplanar,

$$
\begin{aligned}
S_{\mathbf{r}}^{+}= & \sqrt{2 S} e^{i \theta_{+}}\left[\left(\left|\psi_{+Q}\right|+\left|\psi_{-Q}\right|\right) \cos \left(\mathbf{Q} \cdot \mathbf{r}+\theta_{-}\right)\right. \\
& \left.+i\left(\left|\psi_{+Q}\right|-\left|\psi_{-Q}\right|\right) \sin \left(\mathbf{Q} \cdot \mathbf{r}+\theta_{-}\right)\right]
\end{aligned}
$$

The degree of noncoplanarity is controlled by the DM coupling $g$. The distorted-V state has finite longitudinal chirality [recall Eq. (8)], which is proportional to the density imbalance between the $\pm \mathbf{Q}$ condensates,

$$
\kappa_{z} \sim 2 S\left(\left|\psi_{+Q}\right|^{2}-\left|\psi_{-Q}\right|^{2}\right) .
$$

Thus, when the magnetic field orients along the DM vector, the transition from the fully polarized state proceeds in two steps as summarized in the schematic phase diagram of Fig. 9. Initially, at $\mu=-g$, one enters the umbrella state with ordering wave vector $\mathbf{Q}$. This state persists at lower fields until $\mu=g\left(\Gamma_{1}+\right.$ $\left.\Gamma_{2}\right) /\left(\Gamma_{1}-\Gamma_{2}\right)$, where it is replaced by a distorted-V state with condensates at both $\pm \mathbf{Q}$ wave vectors. These findings agree fully with a very different analysis in Sec. IV A, which was carried out for specific values of $D / J$ and $1 / S$. Our results here show that the two phases obtained at high fields near saturation in fact emerge very generally provided DM coupling is weak and quantum fluctuations can be adequately captured perturbatively. 


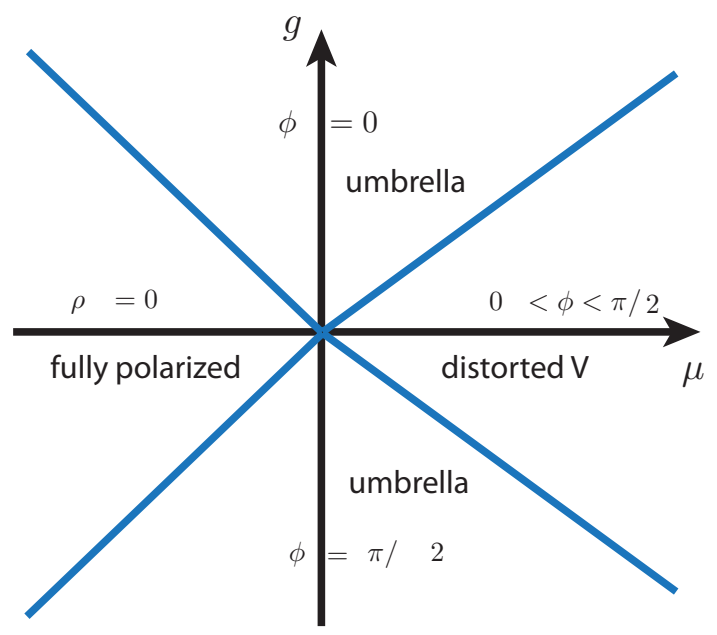

FIG. 9. (Color online) Phase diagram of the model in Eq. (29). The fully polarized state corresponds to $\rho=0$, while all other states exhibit a finite boson density, $\rho>0$. Umbrella states correspond to $\phi=0$ and $\pi / 2$, while the mixed state with $0<\phi<\pi / 2$ represents a distorted-V phase. Observe that the origin of the phase diagram represents a tetracritical point.

\section{Spatially isotropic model with Dzyaloshinskii-Moriya interactions: $J=J^{\prime}, D \neq 0, \mathrm{~h}=h \hat{\mathbf{x}}$}

To apply the formalism developed in this section to the case where the field orients perpendicular to the DM vector, it is simplest to rotate $\mathbf{D}$ and $\mathbf{h}$ such that $\mathbf{h}=h \hat{\mathbf{z}}$ and $\mathbf{D}=D \hat{\mathbf{x}}$. The DM Hamiltonian then reduces to the following combination of three magnon operators,

$$
\begin{aligned}
H_{\mathrm{DM}}= & D \frac{\sqrt{2 S}}{2 i} \sum_{\mathbf{r}, j=1,3}\left[\left(a_{\mathbf{r}}-a_{\mathbf{r}}^{\dagger}\right) a_{\mathbf{r}+\delta_{j}}^{\dagger} a_{\mathbf{r}+\boldsymbol{\delta}_{j}}\right. \\
& \left.-a_{\mathbf{r}}^{\dagger} a_{\mathbf{r}}\left(a_{\mathbf{r}+\delta_{j}}-a_{\mathbf{r}+\delta_{j}}^{\dagger}\right)\right] .
\end{aligned}
$$

Focusing on the condensates, this expression reduces to

$$
\begin{aligned}
H_{\mathrm{DM}}= & D \frac{\sqrt{2 S}}{2 i} \sum_{\mathbf{r}, j=1,3}\left[\psi_{+Q}^{*} \psi_{-Q}\left(\psi_{-Q}-\psi_{+Q}^{*}\right) e^{-i 3 \mathbf{Q} \cdot \mathbf{r}}\right. \\
& \left.\times\left[e^{-i 2 \mathbf{Q} \cdot \boldsymbol{\delta}_{j}}-e^{-i \mathbf{Q} \cdot \boldsymbol{\delta}_{j}}\right]+\{\mathbf{Q} \rightarrow-\mathbf{Q}\}\right] .
\end{aligned}
$$

At this point, commensurability of the three-sublattice spin structure acquires crucial importance, since this implies $e^{ \pm i 3 \mathbf{Q} \cdot \mathbf{r}}=e^{ \pm i 4 \pi x}=1$ for all sites $\mathbf{r}$ of the triangular lattice. The end result, obtained by separating the magnitudes and phases of the condensates using Eq. (30), is rather compact:

$$
H_{\mathrm{DM}}=N 4 \sqrt{6 S} D \rho^{3 / 2} \sin \theta_{+} \sin \left(3 \theta_{-}\right) .
$$

Notice that Eq. (39) is independent of $\phi$. This angle is then determined solely by quantum effects, which select coplanar order, the detailed structure of which is set by the angles $\theta_{ \pm}$. In principle, quantum fluctuations do distinguish the latter angles and favor $\mathrm{V}$ ordering near saturation; this selection is, however, very weak and appears only when one includes higher-order terms in Eq. (23) (involving six boson fields). ${ }^{45}$ In contrast, DM coupling distinguishes these angles more readily. One can immediately observe that, for $D>0$, minimization of Eq. (39) requires that $\theta_{+}= \pm \pi / 2$. Let us choose for concreteness $\theta_{+}=$ $+\pi / 2$. By consulting Eq. (24), one finds that the plane in which spins order is orthogonal to the (rotated) DM vector. That is,

$$
\begin{gathered}
S_{\mathbf{r}}^{x}=0, \\
S_{\mathbf{r}}^{y}=\sqrt{8 S \rho} \cos \left(\mathbf{Q} \cdot \mathbf{r}+\theta_{-}\right) .
\end{gathered}
$$

At the same time, we need to impose $\sin \left(3 \theta_{-}\right)=-1$ given our choice for $\theta_{+}$. The different solutions for $\theta_{-}$simply describe equivalent magnetic structures connected by lattice translations, so we focus for concreteness on $\theta_{-}=\pi / 2$. This choice results in $S_{\mathbf{r}}^{y}=-\sqrt{8 S \rho} \sin (\mathbf{Q} \cdot \mathbf{r})$. For the triangular lattice, the product

$$
\mathbf{Q} \cdot \mathbf{r}=\frac{2 \pi}{3} \nu \bmod 2 \pi
$$

takes on three inequivalent values: $0(\nu=0), 2 \pi / 3(\nu=1)$, and $-2 \pi / 3(v=-1)$. Correspondingly, the ordered $S^{y}$ spin components take on values that are zero $(v=0)$, positive $(v=$ $1)$ and negative $(v=-1)$. This represents the three-sublattice inverted-Y state found previously in Sec. IV B for a specific value of $D / J$ and $1 / S$. The rather different approach adopted here reveals that the onset of inverted-Y order induced by DM coupling near saturation is in fact a very generic conclusion.

\section{Interplay between spatial anisotropy and Dzyaloshinskii-Moriyainteractions: $J \neq J^{\prime}, D \neq 0$}

We will now begin addressing for the first time the influence of spatial anisotropy, which can be incorporated in a particularly simple manner near the saturation field. Let us first address the classical model without DM coupling. When $J \neq J^{\prime}$, the Fourier transform of the exchange interaction (20) is now minimized at generally incommensurate wave vectors $\pm \mathbf{Q}$ given by

$$
\mathbf{Q}=2 \cos ^{-1}\left(\frac{-J^{\prime}}{2 J}\right) \hat{\mathbf{x}} .
$$

Consequently, the difference between $\Gamma_{1,2}$ defined in Eqs. (26) is nonzero already at the classical level; to order $\left(J-J^{\prime}\right)^{2} / J^{2}$, one finds $\Gamma_{1}-\Gamma_{2}=-9\left(J-J^{\prime}\right)^{2} /(2 J)$. It follows from our analysis in Sec. V A that since $\Gamma_{2}>\Gamma_{1}$ here, incommensurate umbrella states are stabilized classically below the saturation field, even with arbitrarily weak exchange anisotropy.

The inclusion of quantum fluctuations changes the situation in an interesting way. Recalling that $\Gamma_{1}>\Gamma_{2}$ due to quantum effects in the isotropic limit, to leading order in $1 / S$ and second order in exchange anisotropy, we have

$$
\Gamma_{1}-\Gamma_{2} \approx \frac{\alpha J}{S}-\frac{9\left(J-J^{\prime}\right)^{2}}{2 J}
$$

for some constant $\alpha$. It is now apparent that the planar ordering favored by quantum fluctuations is in fact stable over a finite range of anisotropy, though due to the shift in $\mathbf{Q}$, such planar configurations will now be incommensurate. Only when the anisotropy reaches a critical strength are these states supplanted by umbrella order.

This analysis can be readily extended to incorporate DM coupling as well. Consider first the field orientation $\mathbf{h}=h \hat{\mathbf{z}}$, where the magnetic field and $\mathbf{D}$ vector are parallel. As long as spatial anisotropy is sufficiently weak, so that $\Gamma_{1}$ exceeds $\Gamma_{2}$, the phase diagram shown in Fig. 9 remains qualitatively 
intact (though the umbrella and distorted-V states become incommensurate). As $\left|J-J^{\prime}\right|$ increases, the incommensurate distorted-V state arises over a progressively smaller region of the phase diagram until, when $\Gamma_{1}=\Gamma_{2}$, it is removed entirely. At larger anisotropy, only incommensurate umbrella phases appear just below saturation.

The case where the magnetic field orients perpendicular to the DM vector is even more straightforward. In the preceding subsection the effectiveness of DM coupling relied critically on the wave vector $\mathbf{Q}$ being commensurate; see Eq. (38). With incommensurate $\mathbf{Q}$, the corresponding term sums to zero and thus drops out. Thus the DM interaction is effectively gone and we then recover the physics discussed above for the anisotropic quantum model without DM interactions.

\section{SPATIALLY ANISOTROPIC MODEL: $J \neq J^{\prime}, D=0$}

\section{A. Classical limit}

In the remaining sections, we will endeavor to address the phase diagram in the presence of spatial anisotropy at arbitrary fields. We start by treating the case without DM interactions for simplicity. Arbitrarily weak anisotropy $J-J^{\prime}$ destroys the accidental classical ground-state degeneracy of the isotropic model in favor of an incommensurate spiral ground state at all fields, similarly to the situation described above near saturation. The ordering wave vector is in fact given by Eq. (42) for all values of the magnetic field below saturation; the corresponding spin state is described by

$$
\mathbf{S}(\mathbf{r})=\sin \theta[\cos (\mathbf{Q} \cdot \mathbf{r}) \hat{\mathbf{x}}+\sin (\mathbf{Q} \cdot \mathbf{r}) \hat{\mathbf{y}}]+\cos \theta \hat{\mathbf{z}} .
$$

While a unique classical ground state therefore emerges for all fields at zero temperature, the finite-temperature phase diagram is much more complicated and interesting. This can be anticipated on physical grounds. Indeed, as a general rule, thermal fluctuations prefer coplanar over noncoplanar states (see the discussion in Sec. III); thus for sufficiently weak anisotropy, one can expect that the entropic gain from planar order should be able to overcome its classical energy cost over a range of temperatures. This means that planar states should appear above some critical temperature $T_{\mathrm{pl}}$. It is reasonable to expect that $T_{\mathrm{pl}} \sim\left(J-J^{\prime}\right)^{2} / J$, since the classical energy gain by umbrella states occurs at second order in $J-J^{\prime}$. It is also clear that as $J-J^{\prime}$ increases, the planar states will be gradually pushed toward higher temperatures and disappear altogether above some critical value (which is magnetic-field dependent) of the spatial anisotropy.

Of all the planar phases considered, the UUD state, which in fact is collinear, yields the highest entropy at finite temperature. The relative stability of the UUD state over the Y and V states is clear already at the spatially isotropic point: Fig. 3 shows that at finite $T$ the collinear state expands at the expense of the planar ones. We thus expect the UUD state to persist the most upon deformation of the model's parameters. Our numerical findings fully support this conclusion and reveal the rather nontrivial phase structure of the classical model. Figure 10 displays the rough phase diagram obtained using classical Monte Carlo with $J^{\prime} / J=0.765$. This value was chosen due to its closeness to the estimate for $\mathrm{Cs}_{2} \mathrm{CuBr}_{4}$, and the fact that for this anisotropy strength, the wave vector $\mathbf{Q}$ fits into the $48 \times 48$

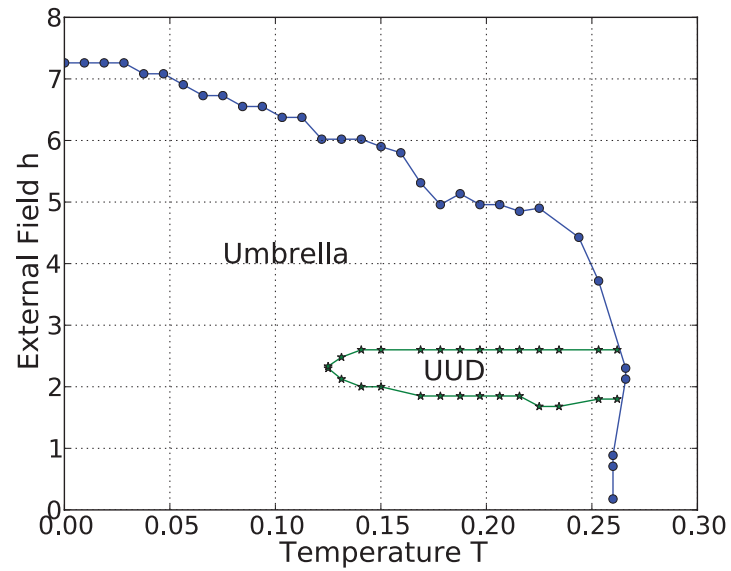

FIG. 10. (Color online) Phase diagram of the classical anisotropic model with $J^{\prime} / J=0.765$. Interestingly, at this anisotropy strength the entropically favored planar phases are absent (within the resolution of our numerics), save for the collinear UUD state, which now requires intermediate temperatures to appear.

system so that incommensurate orders are not frustrated in the geometries we simulated. In these simulations we have taken 20000 Monte Carlo steps for thermalization and 20000 more for measurements.

Remarkably, the UUD state indeed remains and floats above the energetically preferred umbrella phase. The UUD order can be clearly identified by the behavior of $d M / d h$ versus field. As shown in Fig. 11, at an intermediate temperature of $T=0.168 \mathrm{~J}$ we observe a pronounced $\operatorname{dip}$ in $d M / d h$, indicating a diminished slope of the magnetization in the field interval between $h_{c 1}$ and $h_{c 2}$. Of course, slower growth of the magnetization is expected for the magnetization plateau. At a lower temperature of $T=0.12 \mathrm{~J}, d M / d h$ shows no such variation indicating the absence of UUD order. This picture is further corroborated by the temperature dependence of the chirality as Fig. 12 illustrates for $h=2.3 \mathrm{~J}$. One clearly sees a discontinuous jump at $T \approx 0.13 \mathrm{~J}$ in both the transverse and longitudinal chiralities, indicating a first-order finite-temperature transition from the entropy-stabilized UUD state to an umbrella phase. Note that, with the possible

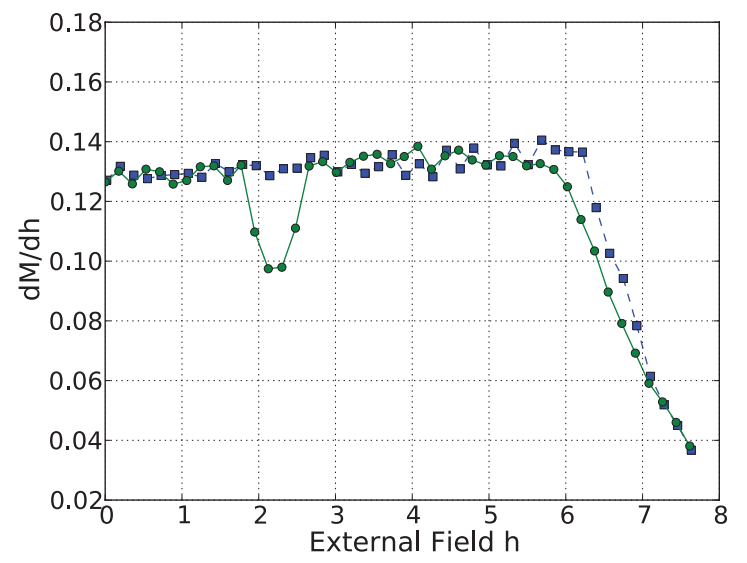

FIG. 11. (Color online) $d M / d h$ versus $h$ at $T=0.168 J$ (green circles) and $T=0.12 J$ (blue squares) for the classical anisotropic model with $J^{\prime} / J=0.765$. 


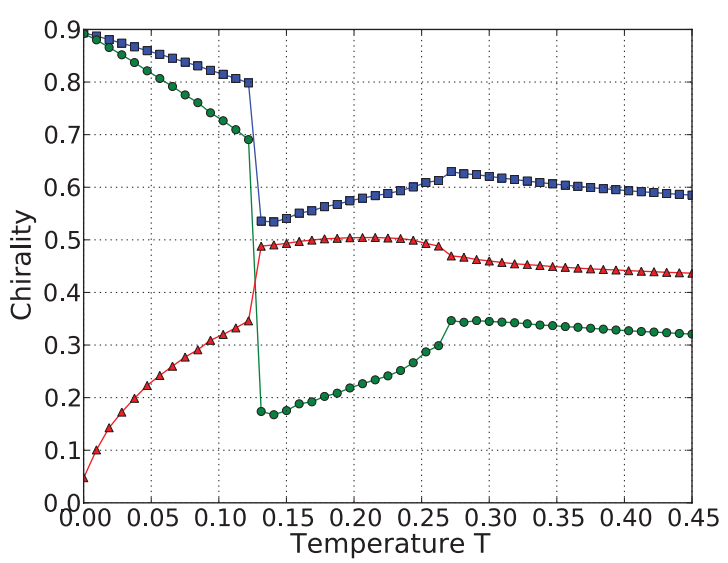

FIG. 12. (Color online) Chiralities $\kappa$ (blue squares), $\kappa_{\perp}$ (red triangles), and $\kappa_{z}$ (green circles) versus $T$ at $h=2.3 J$ for the classical anisotropic model with $J^{\prime} / J=0.765$. The discontinuous jumps near $T=0.13 \mathrm{~J}$ signify a first-order transition between umbrella and UUD phases.

exception of the small regions near the UUD boundary where our numerics do not have enough accuracy to reach any definite conclusions, the Y and V planar states are absent in the phase diagram. At the anisotropy we analyzed here, they are replaced by the energetically favorable umbrella structure.

The already nontrivial phase diagram we obtained here certainly deserves more extensive numerical investigation. In particular, it would be interesting to perform a systematic study increasing $J-J^{\prime}$ from zero to explore the collapse of the Y/UUD/V states and accompanying onset of umbrella order. We note that a similar finite-temperature competition between energetically-favorable and entropically-favorable states has been previously reported in more complex spin systems: frustrated pyrochlore ${ }^{50,51}$ and Shastry-Sutherland antiferromagnets. ${ }^{52}$ It is worth noting that the roots of this behavior can be traced to the famous Pomeranchuk effect in ${ }^{3} \mathrm{He}$ where the crystal phase of ${ }^{3} \mathrm{He}$, which is characterized by exponentially weak in distance exchange interaction between localized spins, has higher entropy than the normal Fermiliquid phase. As a result, upon heating the liquid phase freezes into a solid. ${ }^{53,54}$

\section{B. Pseudoquantum ground states}

Our goal now will be to use Monte Carlo numerics to understand the zero-temperature phase diagram when both exchange anisotropy and quantum effects (modeled via the biquadratic approximation discussed in Sec. III E) are present. Again, to avoid becoming trapped in a local energy minimum, the phase diagram was obtained using simulated annealing as described in Sec. III E.

To explore the competition between anisotropy and quantum effects, simulations were performed with fixed $S=1 / 2$ but numerous anisotropies $J^{\prime} / J=1, \quad 0.95, \quad 0.9, \quad 0.85, \quad 0.8, \quad 0.765$, and 0.7. We used $M, d M / d h, K$, and $\kappa$ to construct the phase diagram at each $J^{\prime} / J$; Fig. 13 summarizes our findings. An interesting feature of the phase diagram is the complete absence of noncoplanar umbrella states, despite the fact that this type of order uniquely minimizes the classical energy at all

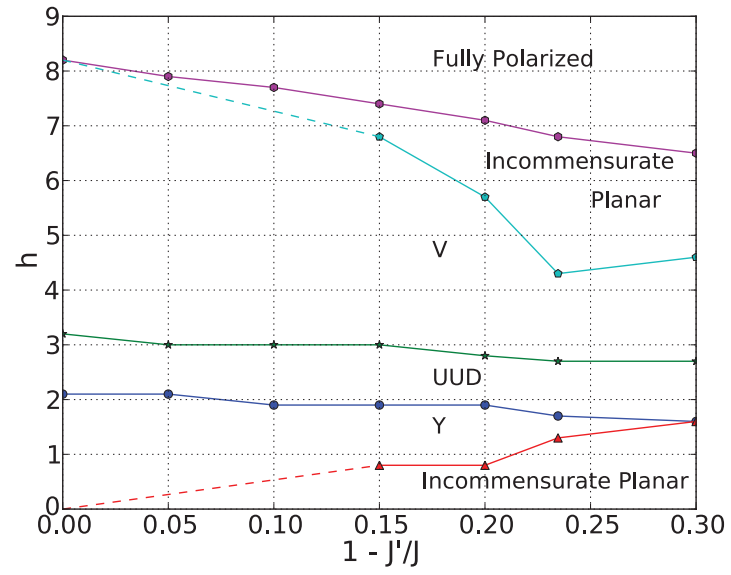

FIG. 13. (Color online) Ground-state phase diagram for the spatially anisotropic triangular antiferromagnet with quantum fluctuations modeled via the biquadratic approximation discussed in Sec. III E. All data points were obtained with Monte Carlo simulated annealing numerics. Dashed lines represent phase boundaries, which are absent due to finite-size effects in our simulations but are expected on general grounds for an infinite system.

fields. Even for rather substantial anisotropy $J-J^{\prime} \sim 0.3 J$, quantum effects (modeled here via the biquadratic interaction) qualitatively alter the magnetization process.

A second interesting feature is the persistence of commensurate Y/UUD/V states despite the exchange anisotropy. The UUD state is particularly stable, and at least within the approximations used is hardly affected by the finite $J-J^{\prime}$ values studied. In its vicinity, the commensurate $\mathrm{Y}$ and $\mathrm{V}$ states appear over a finite field interval, with the latter being more robust than the former against anisotropy. All of this is in agreement with the previous large- $S$ analytical investigation of Ref. 23. Incommensurate planar phases, which reflect a nontrivial compromise between quantum fluctuations and anisotropy, appear at low fields and near saturation; in the thermodynamic limit, these are expected to occupy a progressively larger portion of the phase diagram as anisotropy increases.

It is worth briefly remarking on finite-size effects in our simulations. First, in Fig. 13 the high-field incommensurate phase appears over a broader field range at $J^{\prime} / J=0.765$ than $J^{\prime} / J=0.7$. This artifact arises because the incommensurate spin structure that would appear in an infinite system is less frustrated by the periodic boundary conditions at the former anisotropy strength. Second, we note that incommensurate order sets in at low and high fields only when $J^{\prime} \lesssim 0.85 J$ in our simulations. Closer to the isotropic limit, they are simply absent. This reflects finite-size effects arising from the relatively small systems modeled here. We expect that the phase boundary between the $\mathrm{Y}$ and incommensurate planar states emanates from the $h=0, J^{\prime}=J$ point. Similarly, the phase boundary between the $\mathrm{V}$ and the incommensurate planar states extends all the way to $h=h_{\text {sat }}, J^{\prime}=J$; indeed, in Sec. V D, we found that arbitrarily weak anisotropy is sufficient to produce incommensurate planar order near saturation. These phase lines represent commensurate-incommensurate transitions. We have indicated the expected transitions with dashed lines in Fig. 13. 


\section{SPATIALLY ANISOTROPIC MODEL WITH DZYALOSHINSKII-MORIYA INTERACTIONS:$$
J \neq J^{\prime}, D \neq 0
$$

\section{A. Classical ground states}

Finally, we are in position to consider the full model featuring all of the ingredients we set out to study: spatial anisotropy, DM coupling, and quantum fluctuations. As a first step, we will establish the phase diagram in the classical case. For fields $\mathbf{h}=h \hat{\mathbf{z}}$ directed along the DM vector, the classical ground states simply correspond to the incommensurate umbrella order for all fields up to saturation. This outcome is extremely natural given our earlier findings that for this field orientation both DM coupling and anisotropy separately favor umbrella states.

The phase diagram is more subtle for fields $\mathbf{h}=h \hat{\mathbf{x}}$ oriented perpendicular to the DM vector. We found earlier that spatial anisotropy favors incommensurate umbrella states, while DM interactions prefer inverted $\mathrm{Y}$ order; thus, the resolution of their competition is far from obvious. Focusing on $J^{\prime} / J=0.765$ (again, this value minimizes finite-size effects) and $D / J=0.05$, we find using simulated annealing that the incommensurate coplanar order arises at low fields, $h \lesssim 0.34 h_{\text {sat }}$, reflecting a nontrivial compromise between these competing interactions. DM coupling dominates at intermediate fields $0.34 h_{\text {sat }} \lesssim h \lesssim 0.8 h_{\text {sat }}$, where inverted-Y states appear. The appearance of such a broad commensurate state in the anisotropic system even with only quadratic spin couplings is rather remarkable. Finally, at larger fields up to saturation, spatial anisotropy dominates leading to noncoplanar umbrella order. One can in fact analytically estimate the phase boundaries between these three spin states found in our numerics. The calculation is described in Appendix B, and the resulting classical phase diagram appears in Fig. 14.

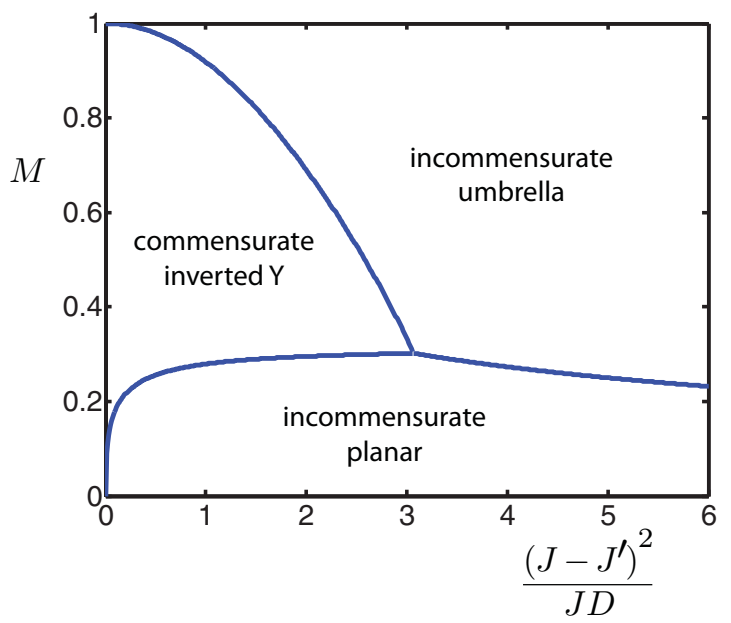

FIG. 14. (Color online) Classical phase diagram for spins with spatially anisotropic exchange interactions, DM coupling, and a magnetic field applied perpendicular to the DM vector. Phase boundaries were computed analytically to leading order in DM strength $D / J$ and anisotropy strength $\left(J-J^{\prime}\right)^{2} / J^{2}$ as described in Appendix B.

\section{B. Pseudoquantum ground states}

Let us now explore how quantum effects_-again modeled within the biquadratic approxiation-modify the classical phase diagrams discussed above. Consider first the $\mathbf{h}=h \hat{\mathbf{z}}$ field orientation. The situation at high fields was already analyzed in Sec. V D, where we found the presence of umbrella order just below saturation, followed by an incommensurate planar state provided anisotropy was not too strong. At intermediate fields, we found in the quantum problem with either DM coupling or spatial anisotropy that commensurate planar phases emerged. It is thus reasonable to anticipate the same outcome when both elements are present, at least for sufficiently weak DM coupling and anisotropy. At low fields, DM coupling led to umbrella order in the quantum problem analyzed in Sec. IV A 3, while the interplay between spatial anisotropy and quantum effects led to an incommensurate planar state in Sec. VIB.

Putting together these findings suggests that the phase diagram for the spatially anisotropic pseudoquantum model depicted in Fig. 13 evolves in the following manner as one increases the DM coupling strength from zero. First, umbrella order immediately begins to "eat away" at the planar phases just below saturation, occupying a progressively larger fraction of the high-field phase diagram as the DM coupling increases. The low-field incommensurate planar state stabilized by the interplay between spatial anisotropy and quantum fluctuations is more robust against DM coupling. Only beyond a critical value of the DM coupling does umbrella order begin to take over in the low-field portion of the phase diagram. Of course, more complicated scenarios are also possible, particularly if DM coupling and/or anisotropy are not especially weak; a detailed study of the problem for this field orientation would be interesting to carry out in future work.

Our main focus, however, is on the low-symmetry field orientation $\mathbf{h}=h \hat{\mathbf{x}}$, where the magnetic field and DM vectors are orthogonal. This is the physical situation relevant for the interesting experiments of Ref. 19, which motivated this study. We explored the zero-temperature phase diagram here using extensive simulating annealing numerics, modeling quantum fluctuations as before using the biquadratic approximation. We note that particular care must be taken when performing these simulations to avoid spurious finite-size effects. In particular, at low fields in $48 \times 48$ systems, we found an unusual incommensurate planar state exhibiting structure-factor peaks at two incommensurate wave vectors with nonzero momentum $Q_{y}$ along the $y$ direction. This phase, however, proved to arise due to finite-size effects-upon increasing the system size to $192 \times 192$, order characterized by a single wave vector and vanishing $Q_{y}$ emerged.

Figure 16 summarizes our results. As noted in Sec. III B, the Hamiltonian no longer possesses any continuous symmetries; hence some of the phase boundaries discussed earlier disappear from the phase diagram and become crossovers. One of these is the transition between the $\mathrm{Y}$ and the UUD states. The difference between these phases in the problem without DM coupling originates from the finite superfluid component of the Y state, present due to spontaneous breaking of U(1) spin rotations about the field axis, along with rotation symmetry that the $\mathrm{Y}$ state breaks but the UUD state does not. In the $\mathbf{D} \perp \mathbf{h}$ 


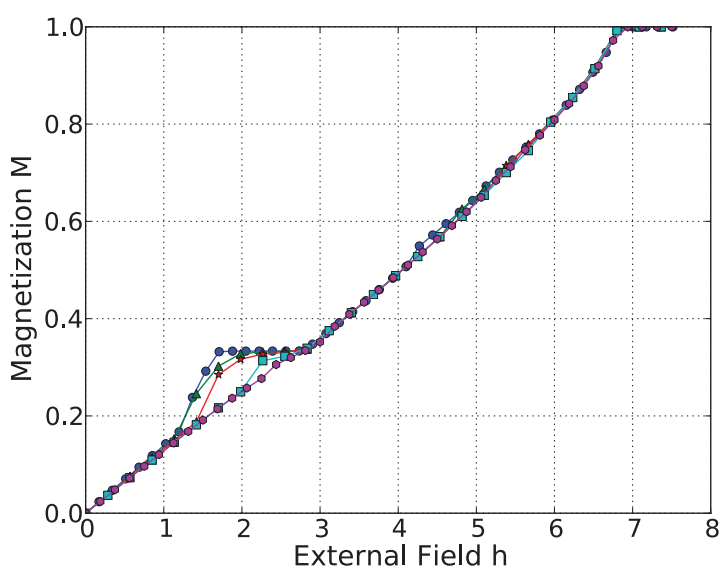

FIG. 15. (Color online) Magnetization vs field at temperature $T=0.001 J$ for the spatially anisotropic antiferromagnet with $J^{\prime}=$ $0.765 \mathrm{~J}, \mathrm{DM}$ interactions satisfying $\mathbf{D} \perp \mathbf{h}$, and quantum effects modeled via a biquadratic interaction. The curves shown correspond to different values of the DM strength: $D=0$ (blue circles), $D=$ $0.01 J$ (green triangles), $D=0.02 J$ (red stars), $D=0.04 J$ (cyan squares), and $D=0.05 J$ (pink hexagons). The quick rounding of the lower edge of the plateau reflects the symmetry equivalence of the $\mathrm{Y}$ and UUD states when DM interactions are present in this field orientation.

problem, these two states are symmetry equivalent as they only break the same discrete lattice symmetries, and are thus not distinct phases. This shows up in our numerical simulations as a quick rounding of the lower end of the magnetization plateau upon increasing the DM coupling at fixed $J^{\prime} / J$, as illustrated in Fig. 15. Simultaneously, for $D$ as small as $D=0.01 \mathrm{~J}$, the coplanarity $K$ becomes finite, although small, inside the former UUD interval between the $\mathrm{Y}$ and $\mathrm{V}$ states.

We also observe persistence of the distorted-V state at intermediate fields above the (former) UUD state. (Note that as discussed in Sec. III B, the symmetry distinction between the distorted-V and UUD orders persists in this field orientation, so that a bona fide phase transition separates these states.) In fact, it appears that the distorted- $\mathrm{V}$ state is the most stable of all the commensurate states considered previously. Because the spins in this phase can smoothly adjust to gain DM energy, this state survives even at the strongest DM coupling strength $D / J=0.05$ considered by us. By contrast, the previously robust UUD state, having lost its symmetry distinction from the less stable $\mathrm{Y}$ state as discussed above, is seen in Fig. 15 to essentially disappear for such a strong DM coupling.

Our simulations also reveal that for sufficiently strong DM coupling $D \gtrsim 0.04 \mathrm{~J}$, a narrow region of the commensurate inverted-Y state appears above the distorted-V phase. This is quite consistent with the phase diagram of Fig. 14 for the classical model described in the previous section; being a prominent phase there, the inverted-Y state is also natural in the quantum problem once quantum effects are sufficiently "weakened" by spatial anisotropy and DM interactions. The transition between the inverted-Y and distorted- $\mathrm{V}$ states appears continuous in our numerics and hard to pin down precisely, in part because the overall extent of this phase is

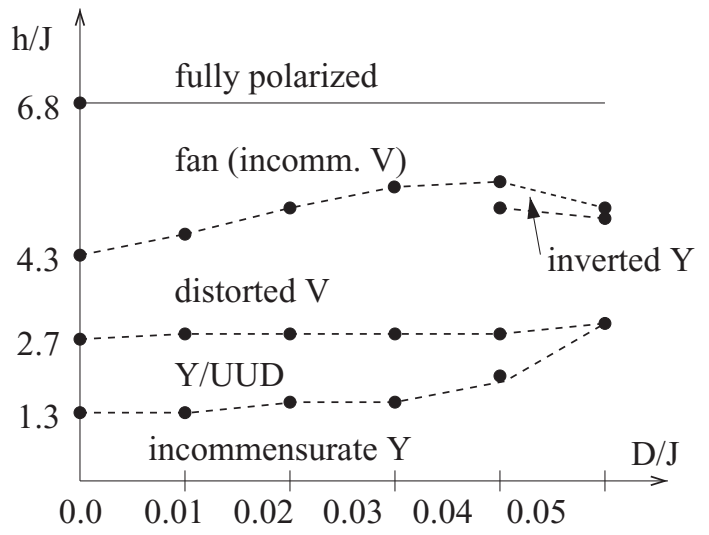

FIG. 16. Sketch of the phase diagram for the quantum spatially anisotropic model with varying DM coupling strength, $J^{\prime} / J=$ 0.765 , and a magnetic field $\mathbf{h}$ oriented perpendicular to the DM vector. Data points indicate phase boundaries determined using simulating annealing numerics with quantum fluctuations modeled via a biquadratic interaction. Dashed lines interpolate between these data points and are drawn for convenience. The location of the inverted-Y phase is approximate as discussed in the main text.

rather narrow. For this reason, its phase boundary in Fig. 16 is less accurate than for the other phases.

The remaining high- and low-field regions are found to be occupied by the incommensurate coplanar states, which owe their stability to quantum fluctuations. This is particularly clear for the high-field region near saturation where an incommensurate analog of the $\mathrm{V}$ state wins over the classical umbrella state only due to $1 / S$ interactions between spin waves, as discussed in Sec. V D.

\section{CONCLUSIONS}

We have explored the phase diagram of a spatially anisotropic triangular lattice quantum antiferromagnet subject to asymmetric DM interactions and an external magnetic field. By treating spatial anisotropy $\left(J-J^{\prime}\right) / J$, quantum fluctuations due to the finite spin value $S$, and DM coupling $D$ as perturbations of the well-understood isotropic classical antiferromagnet, we have found a rich variety of behaviors sensitive to the relative strengths of the perturbations considered. The root of this richness lies in the large accidental degeneracy of the unperturbed model with $J^{\prime}=J, S=\infty$, and $D=0$, along with the fact that each of these perturbations favors different ordered states in the manifold of accidentally degenerate configurations.

Our main findings are as follows. (1) In agreement with numerous previous studies for the isotropic model without DM coupling, we observe that quantum fluctuations select coplanar $\mathrm{Y}$ and $\mathrm{V}$ states and a collinear UUD phase out of infinitely many degenerate states available in the classical limit $S \rightarrow \infty$. Quantum effects most effectively split this degeneracy in the vicinity of one-third magnetization where nearly collinear lowenergy states are accessible. This implies a greater robustness of the quantum-selected states to additional perturbations in these regions of the phase diagram, a trend that is indeed seen throughout our study. We also established that quantum effects can be semiquantitatively modeled within a purely 
classical Hamiltonian by incorporating a biquadratic spin interaction with a field-dependent coupling chosen to mimic $1 / S$ corrections to the classical ground-state energies. Such a purely classical model has the virtue of allowing conventional Monte Carlo simulations to be employed to ascertain the (approximate) phase diagram when all the competing interactions of interest are present.

(2) Adding DM interactions introduces two new commensurate states into consideration-distorted-V and inverted-Y orders. With fields applied perpendicular to the triangular lattice plane, DM coupling stabilizes the umbrella order classically at all fields up to saturation. Quantum effects, however, stabilize a distorted-V state at intermediate fields, bordered on both sides by the classically driven umbrella phase. For all in-plane fields up to saturation, DM interactions select inverted-Y states classically. Quantum fluctuations are still more effective at modifying the phase diagram in this field orientation, producing $\mathrm{Y}$ order at low fields and a distorted-V phase at intermediate fields. Only at high fields does the classically favored inverted-Y state appear in the quantum problem. It is worth pointing out here that the new distorted- $\mathrm{V}$ and inverted- $\mathrm{Y}$ states also appear naturally in the theoretically simple limit of high magnetic fields near saturation, as discussed in Sec. V.

(3) Spatial anisotropy, on the other hand, prefers noncoplanar umbrella (cone) configurations classically. Even this simple classical model, which has a unique ground state, harbors some surprises. We find that for sufficiently small spatial anisotropy, an entropic order-by-disorder selection prevails over energetic considerations over a range of temperatures and magnetic fields. This results in an abrupt first-order phase transition from an umbrella state into a fluctuationstabilized UUD state at finite temperature; see Sec. VIA. This physically reasonable but so far unexplored feature of the classical Heisenberg model with anisotropic exchange interactions deserves more extensive numerical investigation on its own.

(4) The competition between spatial anisotropy and quantum effects (simulated within an effective classical model with biquadratic spin couplings) results in a rich phase diagram shown in Fig. 13. These competing ingredients compromise to form incommensurate versions of the $\mathrm{Y}$ and $\mathrm{V}$ states at low and high fields, respectively. Remarkably, the intermediate-field region of the phase diagram in Fig. 13 is only weakly affected by the anisotropy strengths we analyzed, with the commensurate Y, UUD, and V states favored by quantum effects, all appearing prominently. In particular, the UUD state, which underlies the one-third-magnetization plateau, shows no obvious reduction in its width. All these features of the phase diagram agree well with previous analytical ${ }^{23}$ and numerical ${ }^{27}$ studies.

(5) When spatial anisotropy, DM interactions, and quantum effects (again incorporated in an effective classical Hamiltonian) are all present, we obtain the phase diagram of Fig. 16, which contains most of the phases reviewed above. It is interesting to compare this with the experimental phase diagram of Ref. 19, which features up to nine different phases. Clearly, our phase diagram is less diverse but still contains, for $D \approx 0.04 J$, five different phases, most of which are coplanar. In particular, it is tempting to speculate that one of the puzzling high-field phases (B, III, IV, or $2 / 3$, in the notation of Ref. 19) can be identified with the inverted-Y state appearing near the phase boundary between the distorted-V and the incommensurate planar phases. Further experiments that directly probe the spin structure in the various phases seen are, however, required to make a more definitive comparison with our results.

We emphasize here that nowhere in our study did we find hints of a possible two-thirds-magnetization plateau suggested by several experimental papers. ${ }^{19,24,55}$ In this regard, the possibility of this novel magnetization plateau, at present identified theoretically only once, ${ }^{56}$ requires more careful investigations. It must also be kept in mind that our treatment of quantum fluctuations is only perturbative, and certainly in the $S=1 / 2$ model, at least the phase boundaries will be quantitatively different from what we have found. Whether or not strong quantum fluctuations can induce entirely new states, unseen in our semiclassical analysis, remains an interesting open question.

More broadly, our analysis relies on the observation that near the spatially isotropic point, where the relevant lowenergy states form a three-sublattice pattern, the only relevant DM interaction is that given in Eq. (2). This implies that the phase diagram should be insensitive to the orientation of the magnetic field in the b-c plane of the material. Recent experimental findings support this statement, ${ }^{28}$ and, in our view, should be interpreted as lending strong support to our perturbative approach to $\mathrm{Cs}_{2} \mathrm{CuBr}_{4}$. It is worth pointing out that this is certainly not the case for the isostructural material $\mathrm{Cs}_{2} \mathrm{CuCl}_{4}$; there, it has been established experimentally that phase diagrams for the cases $\mathbf{h} \| \mathbf{b}$ and $\mathbf{h} \| \mathbf{c}$ are indeed different. ${ }^{17}$ That difference has been attributed to additional symmetry-allowed DM interactions ${ }^{18}$ and, fundamentally, is tied to the fact that $\mathrm{Cs}_{2} \mathrm{CuCl}_{4}$ is very much a quasi-one-dimensional material, in contrast to $\mathrm{Cs}_{2} \mathrm{CuBr}_{4}$.

Another, and so far little explored, way to gain insight into the rich physics of frustrated antiferromagnets is provided by a magnetic system's response to various impurities. $\mathrm{Cs}_{2} \mathrm{CuBr}_{4}$ has been probed in this way, ${ }^{24}$ and initial theoretical investigations have appeared recently. ${ }^{57}$

We hope that our study will stimulate further theoretical and experimental studies of $\mathrm{Cs}_{2} \mathrm{CuBr}_{4}$ as well as related materials and models, in the near future.

\section{ACKNOWLEDGMENTS}

We would like to acknowledge helpful conversations and discussions with Leon Balents, Andrey Chubukov, Michel Gingras, Olexei Motrunich, Roderich Moessner, Gil Refael, Yasu Takano, and Mike Zhitomirsky. We are grateful to Yasu Takano for sharing unpublished experimental results with us. We thank the Center for High Performance Computing at the University of Utah for their generous computer time allocation in the initial stages of this project. This research was supported by the National Science Foundation through Grants DMR-0808842 (S.H. and O.A.S.), DMR-1055522 (J.A.), and DGE-0707460 (C.G.) as well as Victoria B. Rodgers and the Rose Hills foundation (C.G.). 


\section{APPENDIX A: SYMMETRY-ALLOWED DZYALOSHINSKII-MORIYA INTERACTIONS IN Cs $\mathrm{CuBr}_{4}$}

The general form of the DM interaction, consistent with crystal symmetry of $\mathrm{Cs}_{2} \mathrm{CuBr}_{4}$, was derived in Ref. 18 for the isostructural material $\mathrm{Cs}_{2} \mathrm{CuCl}_{4}$. For the three-dimensional crystal with layers indexed by $z$, it reads

$$
\begin{aligned}
H_{\mathrm{DM}}= & \sum_{\mathbf{r}}\left(\mathbf{D}^{\prime} \cdot \mathbf{S}_{\mathbf{r}} \times \mathbf{S}_{\mathbf{r}+\delta_{2}}+\mathbf{D}^{+} \cdot \mathbf{S}_{\mathbf{r}} \times \mathbf{S}_{\mathbf{r}+\delta_{1}}\right. \\
& \left.-\mathbf{D}^{-} \cdot \mathbf{S}_{\mathbf{r}} \times \mathbf{S}_{\mathbf{r}-\delta_{3}}\right)
\end{aligned}
$$

where

$$
\begin{gathered}
\mathbf{D}^{\prime}=2 D_{z}(-1)^{z} \hat{\mathbf{z}}+D_{y}(-1)^{y} \hat{\mathbf{y}} \\
\mathbf{D}^{ \pm}=D_{z}^{\prime}(-1)^{z} \hat{\mathbf{z}} \pm D_{x}^{\prime}(-1)^{y+z} \hat{\mathbf{x}}+D_{y}^{\prime}(-1)^{y+z} \hat{\mathbf{y}}
\end{gathered}
$$

We are, however, interested in a 2D triangular antiferromagnet so will henceforth consider only one layer with $z=0$ for concreteness. We now show that for a weakly deformed triangular antiferromagnet, which is an appropriate characterization of $\mathrm{Cs}_{2} \mathrm{CuBr}_{4}$, only two DM couplings, $D_{z}$ and $D_{z}^{\prime}$, need to be retained to leading order in perturbation theory. Furthermore, $D_{z}$ and $D_{z}^{\prime}$ yield first-order contributions, which are, in fact, identical in nature and can thus be combined into a single DM coupling oriented along the crystal $z$ axis.

These results follow from the observation that all classical ground states of the isotropic model form a three-sublattice structure that repeats in the $y$ direction; see Fig. 1. Let us first consider the term $D_{y}$. Because of the repeating structure in the $y$ direction, we see that the cross products of spins from the $A, B$, and $C$ sublattices cancel out due to the oscillating $(-1)^{y}$ factor in the $D_{y}$ term. Thus $D_{y}$ can only appear at second order or higher in perturbation theory, and for this reason can be neglected. Similar reasoning dictates that $D_{x}^{\prime}$ and $D_{y}^{\prime}$, which also include oscillating $(-1)^{y}$ factors, do not contribute to first order and can thus be omitted as well.

Consider now the remaining terms, $D_{z}$ and $D_{z}^{\prime}$. From Fig. 1, we see that $\mathbf{S}_{\mathbf{r}+\delta_{1}}$ and $\mathbf{S}_{\mathbf{r}-\delta_{2}}$ correspond to the same sublattice. Likewise, spins $\mathbf{S}_{\mathbf{r}+\boldsymbol{\delta}_{2}}$ and $\mathbf{S}_{\mathbf{r}-\boldsymbol{\delta}_{3}}$ can also be identified. Consequently, the most general DM Hamiltonian above, when evaluated in an arbitrary three-sublattice classical ground state, reduces to the following expression:

$$
\begin{aligned}
H_{\mathrm{DM}} \rightarrow & \sum_{\mathbf{r}} \hat{\mathbf{z}} \cdot\left(2 D_{z} \mathbf{S}_{\mathbf{r}} \times \mathbf{S}_{\mathbf{r}+\boldsymbol{\delta}_{2}}+D_{z}^{\prime} \mathbf{S}_{\mathbf{r}} \times \mathbf{S}_{\mathbf{r}+\boldsymbol{\delta}_{1}}\right. \\
& \left.-D_{z}^{\prime} \mathbf{S}_{\mathbf{r}} \times \mathbf{S}_{\mathbf{r}-\boldsymbol{\delta}_{3}}\right) \\
= & \sum_{\mathbf{r}} \hat{\mathbf{z}} \cdot\left[\left(D_{z} \mathbf{S}_{\mathbf{r}} \times \mathbf{S}_{\mathbf{r}+\boldsymbol{\delta}_{2}}-D_{z}^{\prime} \mathbf{S}_{\mathbf{r}} \times \mathbf{S}_{\mathbf{r}-\boldsymbol{\delta}_{3}}\right)\right. \\
& \left.-\left(D_{z} \mathbf{S}_{\mathbf{r}} \times \mathbf{S}_{\mathbf{r}-\boldsymbol{\delta}_{2}}-D_{z}^{\prime} \mathbf{S}_{\mathbf{r}} \times \mathbf{S}_{\mathbf{r}+\boldsymbol{\delta}_{1}}\right)\right] \\
\rightarrow & \left(D_{z}-D_{z}^{\prime}\right) \sum_{\mathbf{r}} \hat{\mathbf{z}} \cdot\left(\mathbf{S}_{\mathbf{r}} \times \mathbf{S}_{\mathbf{r}-\boldsymbol{\delta}_{3}}-\mathbf{S}_{\mathbf{r}} \times \mathbf{S}_{\mathbf{r}+\boldsymbol{\delta}_{1}}\right) \\
= & \left(D_{z}^{\prime}-D_{z}\right) \sum_{\mathbf{r}} \hat{\mathbf{z}} \cdot\left[\mathbf{S}_{\mathbf{r}} \times\left(\mathbf{S}_{\mathbf{r}+\boldsymbol{\delta}_{1}}+\mathbf{S}_{\mathbf{r}+\boldsymbol{\delta}_{3}}\right)\right] .
\end{aligned}
$$

Hence, defining $\mathbf{D}=\left(D_{z}-D_{z}^{\prime}\right) \hat{\mathbf{z}} \equiv D \hat{\mathbf{z}}$, we arrive at the DM Hamiltonian (2).

\section{APPENDIX B: ANALYTICAL DETERMINATION OF PHASE BOUNDARIES IN FIG. 14}

In this Appendix, we will sketch the derivation of the phase boundaries depicted in Fig. 14. We adopt a variational approach and calculate the energies for the three phases found in simulations - incommensurate planar, commensurate inverted-Y, and incommensurate umbrella states-to determine which minimizes the energy as a function of field. Of course, there is no guarantee that only these three phases are relevant, but our numerical findings suggest that this is the case for weak anisotropy and DM coupling.

For concreteness, let us take the field in the $y$ direction. For the incommensurate planar state, we work at low fields and parametrize the spins as

$$
\mathbf{S}_{\mathbf{r}}=\cos [\mathbf{Q} \cdot \mathbf{r}+\phi(\mathbf{r})] \hat{\mathbf{x}}+\sin [\mathbf{Q} \cdot \mathbf{r}+\phi(\mathbf{r})] \hat{\mathbf{y}},
$$

where $\mathbf{Q}$ is the incommensurate wave vector from Eq. (42). With $\phi(\mathbf{r})=0$, this expression yields the exact classical ground state at zero field and in the absence of DM coupling. [We will neglect the dependence of $\mathbf{Q}$ on the DM coupling here, which results in energy corrections of order $(D / J)^{2}$.] In finite fields, the function $\phi(\mathbf{r})$ becomes nonzero and gives rise to a net magnetization along the $y$ direction; it suffices to take

$$
\phi(\mathbf{r})=2 M \cos \mathbf{Q} \cdot \mathbf{r} .
$$

Up to order $h^{2}, M$ represents the magnetization, which is given by

$$
M=\frac{h J^{3}}{\left(2 J+J^{\prime}\right)\left[4 J^{3}+J^{\prime 3}-2 J^{2}\left(J^{\prime}-D \sqrt{4-\left(J^{\prime} / J\right)^{2}}\right)\right]} .
$$

(This expression can be obtained by evaluating the energy to second order in $h$, and then minimizing the expression to find M.) The incommensurate planar energy per site to order $h^{2}$, is then

$$
\begin{aligned}
\frac{E_{\text {planar }}}{N}= & -\left\{h M+\frac{D}{J} \sqrt{4-\left(J^{\prime} / J\right)^{2}}\left[J-\left(2 J+J^{\prime}\right) M^{2}\right]\right. \\
& \left.+\frac{2 J^{2}+J^{\prime 2}}{2 J}-\frac{\left(2 J+J^{\prime}\right)^{2}\left(2 J^{2}-2 J J^{\prime}+J^{\prime 2}\right) M^{2}}{2 J^{3}}\right\} .
\end{aligned}
$$

Both the inverted-Y and umbrella states can be easily found at arbitrary fields. For the inverted-Y state, we have

$$
\begin{gathered}
\mathbf{S}_{A}=\hat{\mathbf{y}}, \\
\mathbf{S}_{B}=\sqrt{1-\frac{1}{4}(3 M-1)^{2}} \hat{\mathbf{x}}+\frac{1}{2}(3 M-1) \hat{\mathbf{y}}, \\
\mathbf{S}_{C}=-\sqrt{1-\frac{1}{4}(3 M-1)^{2}} \hat{\mathbf{x}}+\frac{1}{2}(3 M-1) \hat{\mathbf{y}},
\end{gathered}
$$

which yields an energy per site

$$
\begin{aligned}
\frac{E_{\text {inverted-Y }}}{N}= & -\left[\frac{1}{2}\left(J+2 J^{\prime}\right)+h M-\frac{3}{2}\left(J+2 J^{\prime}\right) M^{2}\right. \\
& \left.+D \sqrt{3(1+3 M)}(1-M)^{3 / 2}\right] .
\end{aligned}
$$


The spin configuration for the incommensurate umbrella state is (again ignoring renormalization of $\mathbf{Q}$ by $D$ ) given by Eq. (44); the corresponding energy per site is

$$
\frac{E_{\mathrm{umb}}}{N}=-\left[\frac{2 J^{2}+J^{\prime 2}}{2 J}+h M-\frac{\left(2 J+J^{\prime}\right)^{2}}{2 J} M^{2}\right] .
$$

To leading order in $D / J$ and $\left(J-J^{\prime}\right)^{2} / J^{2}$, the incommensurate planar and inverted-Y state energies cross at a magnetization $M_{1}$ satisfying

$$
\begin{aligned}
& \frac{\left(J-J^{\prime}\right)^{2}}{2 J}\left(1-10 M_{1}^{2}\right) \\
& =\sqrt{3} D\left[\left(1-M_{1}\right)^{3 / 2} \sqrt{1+3 M_{1}}-1+3 M_{1}^{2}\right] .
\end{aligned}
$$

For $M<M_{1}$, the planar state has lower variational energy, while for $M>M_{1}$ the inverted-Y state wins. Taking $D / J=0.05$ and $J^{\prime} / J=0.7$, we find $M_{1} \approx 0.3$. Although our calculation of the planar energy was perturbative in the field and is thus most reliable near $M=0$, this approximate result for $M_{1}$ agrees remarkably well with the lower phase boundary determined numerically for these parameter values.
The umbrella state and inverted-Y energies balance at a magnetization $M_{2}$, which satisfies

$$
\frac{\left(J-J^{\prime}\right)^{2}}{2 J}\left(1+M_{2}\right)=D \sqrt{3\left(1+3 M_{2}\right)\left(1-M_{2}\right)} .
$$

In this case, the inverted-Y state yields a lower energy for $M<M_{2}$. With $J^{\prime} / J=0.7$ and $D / J=0.05$ we obtain $M_{2} \approx$ 0.7 , also in excellent agreement with numerics. At a critical anisotropy strength $\left(J-J^{\prime}\right)^{2} / J \approx 3 D$, the magnetization values $M_{1}$ and $M_{2}$ coincide, and for larger anisotropy the inverted-Y state no longer appears in the phase diagram. We then have only two phases which compete-the incommensurate planar and umbrella states. To leading order in $D / J$ and $\left(J-J^{\prime}\right)^{2} / J^{2}$, their energies cross at a magnetization

$$
M_{3}=\left[\frac{9\left(J-J^{\prime}\right)^{2}}{2 \sqrt{3} J D}+3\right]^{-1 / 2} .
$$

For $M>M_{3}$, the incommensurate umbrella phase emerges, whereas for $M<M_{3}$ incommensurate planar order appears. Plotting the phase boundaries $M_{1,2,3}$ derived above leads to the classical phase diagram of Fig. 14.
${ }^{1}$ G. H. Wannier, Phys. Rev. 79, 357 (1950).

${ }^{2}$ P. Anderson, Mater. Res. Bull. 8, 153 (1973).

${ }^{3}$ P. Fazekas and P. W. Anderson, Philos. Mag. 30, 423 (1974).

${ }^{4}$ D. A. Huse and V. Elser, Phys. Rev. Lett. 60, 2531 (1988).

${ }^{5}$ B. Bernu, C. Lhuillier, and L. Pierre, Phys. Rev. Lett. 69, 2590 (1992).

${ }^{6}$ L. Capriotti, A. E. Trumper, and S. Sorella, Phys. Rev. Lett. 82, 3899 (1999).

${ }^{7}$ R. Moessner and S. L. Sondhi, Phys. Rev. Lett. 86, 1881 (2001).

${ }^{8}$ H. Kawamura and S. Miyashita, J. Phys. Soc. Jpn. 53, 4138 (1984).

${ }^{9}$ A. V. Chubukov and D. I. Golosov, J. Phys. Condens. Matter 3, 69 (1991).

${ }^{10}$ E. F. Shender, Sov. Phys. JETP 56, 178 (1982).

${ }^{11}$ C. L. Henley, Phys. Rev. Lett. 62, 2056 (1989).

${ }^{12}$ K. Okunishi and T. Tonegawa, J. Phys. Soc. Jpn. 72, 479 (2003).

${ }^{13}$ T. Hikihara, T. Momoi, A. Furusaki, and H. Kawamura, Phys. Rev. B 81, 224433 (2010).

${ }^{14}$ L. E. Svistov, A. I. Smirnov, L. A. Prozorova, O. A. Petrenko, A. Micheler, N. Büttgen, A. Y. Shapiro, and L. N. Demianets, Phys. Rev. B 74, 024412 (2006).

${ }^{15}$ R. Coldea, D. A. Tennant, K. Habicht, P. Smeibidl, C. Wolters, and Z. Tylczynski, Phys. Rev. Lett. 88, 137203 (2002).

${ }^{16}$ R. Coldea, D. A. Tennant, and Z. Tylczynski, Phys. Rev. B 68, 134424 (2003).

${ }^{17}$ Y. Tokiwa, T. Radu, R. Coldea, H. Wilhelm, Z. Tylczynski, and F. Steglich, Phys. Rev. B 73, 134414 (2006).

${ }^{18}$ O. A. Starykh, H. Katsura, and L. Balents, Phys. Rev. B 82, 014421 (2010).

${ }^{19}$ N. A. Fortune, S. T. Hannahs, Y. Yoshida, T. E. Sherline, T. Ono, H. Tanaka, and Y. Takano, Phys. Rev. Lett. 102, 257201 (2009).

${ }^{20}$ T. Ono, H. Tanaka, H. Aruga Katori, F. Ishikawa, H. Mitamura, and T. Goto, Phys. Rev. B 67, 104431 (2003).

${ }^{21}$ H. Tsujii, C. R. Rotundu, T. Ono, H. Tanaka, B. Andraka, K. Ingersent, and Y. Takano, Phys. Rev. B 76, 060406 (2007).
${ }^{22}$ Y. Fujii, H. Hashimoto, Y. Yasuda, H. Kikuchi, M. Chiba, S. Matsubara, and M. Takigawa, J. Phys. Condens. Matter 19, 145237 (2007).

${ }^{23}$ J. Alicea, A. V. Chubukov, and O. A. Starykh, Phys. Rev. Lett. 102, 137201 (2009).

${ }^{24}$ T. Ono et al., J. Phys. Soc. Jpn.74S, 135 (2005).

${ }^{25}$ K. Foyevtsova, I. Opahle, Y.-Z. Zhang, H. O. Jeschke, and R. Valentí, Phys. Rev. B 83, 125126 (2011).

${ }^{26}$ R. S. Gekht and I. N. Bondarenko, J. Exp. Theor. Phys. 84, 345 (1997).

${ }^{27}$ T. Tay and O. I. Motrunich, Phys. Rev. B 81, 165116 (2010).

${ }^{28}$ Y. Takano (private communication).

${ }^{29}$ D. Heidarian and K. Damle, Phys. Rev. Lett. 95, 127206 (2005).

${ }^{30}$ H. C. Jiang, M. Q. Weng, Z. Y. Weng, D. N. Sheng, and L. Balents, Phys. Rev. B 79, 020409 (2009).

${ }^{31}$ F. Wang, F. Pollmann, and A. Vishwanath, Phys. Rev. Lett. 102, 017203 (2009).

${ }^{32}$ A. Albuquerque et al., J. Magn. Magn. Mater. 310, 1187 (2007).

${ }^{33}$ S. Watarai, S. Miyashita, and H. Shiba, J. Phys. Soc. Jpn. 70, 532 (2001).

${ }^{34}$ M. Gvozdikova, P. Melchy, and M. Zhitomirsky, J. Phys. Condens. Matter 23, 164209 (2011).

${ }^{35}$ M. Wintel, H. U. Everts, and W. Apel, Phys. Rev. B 52, 13480 (1995).

${ }^{36}$ H. Kawamura and A. Yamamoto, J. Phys. Soc. Jpn. 76, 073704 (2007).

${ }^{37}$ P.-E. Melchy and M. E. Zhitomirsky, Phys. Rev. B 80, 064411 (2009).

${ }^{38}$ H. Kawamura, A. Yamamoto, and T. Okubo, J. Phys. Soc. Jpn. 79, 023701 (2010).

${ }^{39}$ T. Okubo and H. Kawamura, J. Phys. Soc. Jpn. 79, 084706 (2010).

${ }^{40}$ L. Seabra, T. Momoi, P. Sindzingre, and N. Shannon, e-print arXiv:1109.2211 (unpublished).

${ }^{41}$ T. Nikuni and H. Shiba, J. Phys. Soc. Jpn. 62, 3268 (1993). 
${ }^{42}$ M. E. Zhitomirsky and T. Nikuni, Phys. Rev. B 57, 5013 (1998).

${ }^{43}$ C. L. Henley, Can. J. Phys. 79, 1307 (2001).

${ }^{44}$ E. S. Batyev and L. S. Braginskii, Sov. Phys. JETP 60, 781 (1984).

${ }^{45}$ T. Nikuni and H. Shiba, J. Phys. Soc. Jpn. 64, 3471 (1995).

${ }^{46}$ M. Y. Veillette, J. T. Chalker, and R. Coldea, Phys. Rev. B 71, 214426 (2005).

${ }^{47}$ T. Giamarchi, C. Ruegg, and O. Tchernyshyov, Nat. Phys. 4, 198 (2008).

${ }^{48}$ H. T. Ueda and K. Totsuka, Phys. Rev. B 80, 014417 (2009).

${ }^{49}$ A. D. Bruce and A. Aharony, Phys. Rev. B 11, 478 (1975).

${ }^{50}$ C. Pinettes, B. Canals, and C. Lacroix, Phys. Rev. B 66, 024422 (2002).
${ }^{51}$ G.-W. Chern, R. Moessner, and O. Tchernyshyov, Phys. Rev. B 78, 144418 (2008)

${ }^{52}$ M. Moliner, D. C. Cabra, A. Honecker, P. Pujol, and F. Stauffer, Phys. Rev. B 79, 144401 (2009).

${ }^{53}$ I. Pomeranchuk, Zh. Eksp. Teor. Fiz. 20, 919 (1950).

${ }^{54}$ R. C. Richardson, Rev. Mod. Phys. 69, 683 (1997).

${ }^{55}$ T. Ono et al., J. Phys. Condens. Matter 16, S773 (2004).

${ }^{56}$ S. Miyahara, K. Ogino, and N. Furukawa, Physica B: Condensed Matter 378-380, 587 (2006).

${ }^{57}$ A. Wollny, L. Fritz, and M. Vojta, Phys. Rev. Lett. 107, 137204 (2011). 